Louisiana State University

LSU Digital Commons

$5-1-2020$

\title{
Planet Hunters Tess I: TOI 813, a subgiant hosting a transiting Saturn-sized planet on an 84-day orbit
}

\author{
N. L. Eisner \\ University of Oxford \\ O. Barragán \\ University of Oxford \\ S. Aigrain \\ University of Oxford \\ C. Lintott \\ University of Oxford \\ G. Miller \\ University of Oxford
}

See next page for additional authors

Follow this and additional works at: https://digitalcommons.Isu.edu/physics_astronomy_pubs

\section{Recommended Citation}

Eisner, N., Barragán, O., Aigrain, S., Lintott, C., Miller, G., Zicher, N., Boyajian, T., Briceño, C., Bryant, E., Christiansen, J., Feinstein, A., Flor-Torres, L., Fridlund, M., Gandolfi, D., Gilbert, J., Guerrero, N., Jenkins, J., Jones, K., Kristiansen, M., Vanderburg, A., Law, N., López-Sánchez, A., Mann, A., Safron, E., Schwamb, M., Stassun, K., Osborn, H., Wang, J., Zic, A., Ziegler, C., Barnet, F., Bean, S., \& Bundy, D. (2020). Planet Hunters Tess I: TOI 813, a subgiant hosting a transiting Saturn-sized planet on an 84-day orbit. Monthly Notices of the Royal Astronomical Society, 494 (1), 750-763. https://doi.org/10.1093/mnras/staa138

This Article is brought to you for free and open access by the Department of Physics \& Astronomy at LSU Digital Commons. It has been accepted for inclusion in Faculty Publications by an authorized administrator of LSU Digital Commons. For more information, please contact ir@lsu.edu. 


\section{Authors}

N. L. Eisner, O. Barragán, S. Aigrain, C. Lintott, G. Miller, N. Zicher, T. S. Boyajian, C. Briceño, E. M. Bryant, J. L. Christiansen, A. D. Feinstein, L. M. Flor-Torres, M. Fridlund, D. Gandolfi, J. Gilbert, N. Guerrero, J. M. Jenkins, K. Jones, M. H. Kristiansen, A. Vanderburg, N. Law, A. R. López-Sánchez, A. W. Mann, E. J. Safron, M. E. Schwamb, K. G. Stassun, H. P. Osborn, J. Wang, A. Zic, C. Ziegler, F. Barnet, S. J. Bean, and D. M. Bundy 


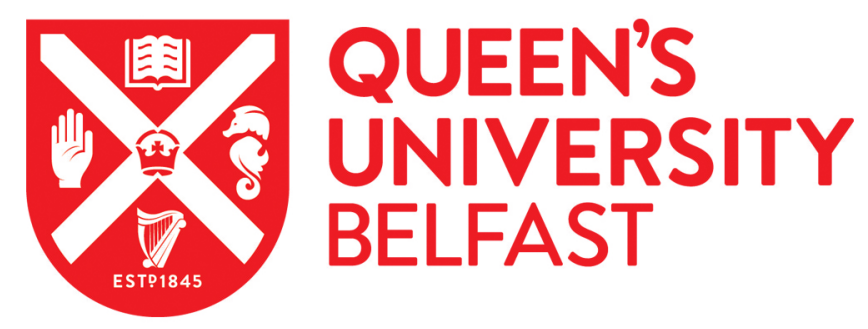

\section{Planet Hunters TESS I: TOI 813, a subgiant hosting a transiting Saturn-sized planet on an 84-day orbit}

Eisner, N. L., Barragán, O., Aigrain, S., Lintott, C., Miller, G., Zicher, N., Boyajian, T. S., Briceño, C., Bryant, E. M., Christiansen, J. L., Feinstein, A. D., Flor-Torres, L. M., Fridlund, M., Gandolfi, D., Gilbert, J., Guerrero, N., Jenkins, J. M., Jones, K., Kristiansen, M. H., ... Tsymbal, A. (2020). Planet Hunters TESS I: TOI 813, a subgiant hosting a transiting Saturn-sized planet on an 84-day orbit. Monthly Notices of the Royal Astronomical Society, 494(1), 750-763. https://doi.org/10.1093/mnras/staa138

Published in:

Monthly Notices of the Royal Astronomical Society

Document Version:

Publisher's PDF, also known as Version of record

Queen's University Belfast - Research Portal:

Link to publication record in Queen's University Belfast Research Portal

Publisher rights

(C) 2020 The Author(s). Published by Oxford University Press on behalf of the Royal Astronomical Society. This work is made available online in accordance with the publisher's policies. Please refer to any applicable terms of use of the publisher.

\section{General rights}

Copyright for the publications made accessible via the Queen's University Belfast Research Portal is retained by the author(s) and / or other copyright owners and it is a condition of accessing these publications that users recognise and abide by the legal requirements associated with these rights.

Take down policy

The Research Portal is Queen's institutional repository that provides access to Queen's research output. Every effort has been made to ensure that content in the Research Portal does not infringe any person's rights, or applicable UK laws. If you discover content in the Research Portal that you believe breaches copyright or violates any law, please contact openaccess@qub.ac.uk. 


\title{
Planet Hunters TESS I: TOI 813, a subgiant hosting a transiting Saturn-sized planet on an 84-day orbit
}

\author{
N. L. Eisner ${ }^{\oplus}{ }^{1 \star}$ O. Barragán, ${ }^{1 \star}$ S. Aigrain, ${ }^{1}$ C. Lintott ${ }^{\oplus},{ }^{1}$ G. Miller, ${ }^{1}$ N. Zicher, ${ }^{1}$ \\ T. S. Boyajian, ${ }^{2}$ C. Briceño, ${ }^{3}$ E. M. Bryant, ${ }^{4,5}$ J. L. Christiansen, ${ }^{6}$ A. D. Feinstein, ${ }^{7}$ \\ L. M. Flor-Torres ${ }^{8}$ M. Fridlund,,${ }^{9,10}$ D. Gandolfi, ${ }^{11}$ J. Gilbert ${ }^{\odot},{ }^{12}$ N. Guerrero, ${ }^{13}$ \\ J. M. Jenkins, ${ }^{6}$ K. Jones,${ }^{1}$ M. H. Kristiansen, ${ }^{14}$ A. Vanderburg, ${ }^{15}$ N. Law, ${ }^{16}$ \\ A. R. López-Sánchez, ${ }^{17,18}$ A. W. Mann, ${ }^{16}$ E. J. Safron, ${ }^{2}$ M. E. Schwamb, ${ }^{19,20}$ \\ K. G. Stassun ${ }^{\odot},{ }^{21,22}$ H. P. Osborn ${ }^{\oplus},{ }^{23}$ J. Wang,${ }^{24}$ A. Zic ${ }^{\oplus},{ }^{25,26}$ C. Ziegler, ${ }^{27}$ F. Barnet,${ }^{28} \dagger$ \\ S. J. Bean,${ }^{28} \dagger$ D. M. Bundy, ${ }^{28} \dagger$ Z. Chetnik, ${ }^{28} \dagger$ J. L. Dawson, ${ }^{28} \dagger$ J. Garstone,${ }^{28} \dagger$ \\ A. G. Stenner, ${ }^{28} \dagger$ M. Huten, ${ }^{28} \dagger$ S. Larish, ${ }^{28} \dagger$ L. D. Melanson, ${ }^{28} \dagger$ T. Mitchell, ${ }^{28} \dagger$ \\ C. Moore,${ }^{28} \dagger$ K. Peltsch,${ }^{28} \dagger$ D. J. Rogers,${ }^{28} \dagger$ C. Schuster,${ }^{28} \dagger$ D. S. Smith,${ }^{28} \dagger$ \\ D. J. Simister, ${ }^{28} \dagger$ C. Tanner, ${ }^{28} \dagger$ I. Terentev ${ }^{28} \dagger$ and A. Tsymbal ${ }^{28} \dagger$
}

Affiliations are listed at the end of the paper

Accepted 2020 January 6. Received 2019 December 20; in original form 2019 September 12

\begin{abstract}
We report on the discovery and validation of TOI $813 \mathrm{~b}$ (TIC $55525572 \mathrm{~b}$ ), a transiting exoplanet identified by citizen scientists in data from NASA's Transiting Exoplanet Survey Satellite (TESS) and the first planet discovered by the Planet Hunters TESS project. The host star is a bright $(V=10.3 \mathrm{mag})$ subgiant $\left(R_{\star}=1.94 R_{\odot}, M_{\star}=1.32 M_{\odot}\right)$. It was observed almost continuously by TESS during its first year of operations, during which time four individual transit events were detected. The candidate passed all the standard light curve-based vetting checks, and ground-based follow-up spectroscopy and speckle imaging enabled us to place an upper limit of $2 M_{\text {Jup }}$ ( 99 percent confidence) on the mass of the companion, and to statistically validate its planetary nature. Detailed modelling of the transits yields a period of $83.8911_{-0.0031}^{+0.0027} \mathrm{~d}$, a planet radius of $6.71 \pm 0.38 R_{\oplus}$ and a semimajor axis of $0.423_{-0.037}^{+0.031} \mathrm{AU}$. The planet's orbital period combined with the evolved nature of the host star places this object in a relatively underexplored region of parameter space. We estimate that TOI $813 \mathrm{~b}$ induces a reflex motion in its host star with a semi-amplitude of $\sim 6 \mathrm{~m} \mathrm{~s}^{-1}$, making this a promising system to measure the mass of a relatively long-period transiting planet.
\end{abstract}

Key words: methods: statistical-planets and satellites: detection-stars: fundamental parameters - stars: individual (TIC-55525572 - TOI 813).

\section{INTRODUCTION}

The Transiting Exoplanet Survey Satellite (TESS; Ricker et al. 2015) is the first nearly all-sky space-based transit search mission. Over the course of its two-year nominal mission, TESS will observe 85 per cent of the sky, split up into 26 observational sectors (13 per

^E-mail: nora.eisner@new.ox.ac.uk (NLE); oscar.barraganvillanueva@physics.ox.ac.uk (OB)

$†$ Citizen Scientist, Zooniverse. hemisphere) that extend from the ecliptic pole to near the ecliptic plane. Targets located at low ecliptic latitudes (around 63 percent of the sky) will be monitored for $\approx 27.4$ continuous days, while a total of $\sim 2$ per cent of the sky at the ecliptic poles will be observed continuously for $\sim 356 \mathrm{~d}$. This observational strategy means that TESS will provide us with a plethora of short period planets $(\lesssim 20 \mathrm{~d})$ around bright ( $V \lesssim 11 \mathrm{mag}$ ), nearby stars, which will allow for detailed characterization (e.g. Barclay, Pepper \& Quintana 2018; Gandolfi et al. 2018; Huang et al. 2018; Esposito et al. 2019).

Longer period planets will, however, be significantly more difficult to detect. This is partly because the transiting probability 
of a planet decreases with increasing orbital distance from the star and partly because detection pipelines typically require two or more transit events in order to gain the signal-to-noise ratio (SNR) needed for detection and often three or more events to confirm a periodicity. The requirement of multiple transit events, in particular, poses a problem for the automated detection of long-period planets in the TESS light curves. This is because only the targets close to the poles will be monitored across multiple observational sectors and will thus have light curves with longer observational baselines and the opportunity to detect longer period planets with multiple transit events. This is reflected in the early TESS results: ${ }^{1} 88$ per cent of the first 1075 TESS Objects of Interest (TOI) have periods $<15 \mathrm{~d}$ and 95 per cent have periods $<30 \mathrm{~d}$. Non-standard methods, such as machine learning (e.g. Pearson, Palafox \& Griffith 2018; Zucker \& Giryes 2018), probabilistic transit model comparison (e.g. ForemanMackey et al. 2016), or citizen science (e.g. Fischer et al. 2012), can in some cases outperform standard transit search pipelines for longer period planets, and are often sensitive to single transit events that the pipelines routinely ignore. This motivated us to initiate systematic searches for transits in the TESS data using some of these alternative methods.

In this paper, we announce the detection and statistical validation of TOI 813 b (TIC 55525572 b), a Saturn-sized planet orbiting around a bright subgiant star. The candidate was initially identified as a single-transit event by citizen scientists taking part in the Planet Hunters TESS (PHT) project. ${ }^{2}$ The later detection of further transit events allowed us to constrain the orbital period to $\sim 84 \mathrm{~d}$, making it, to the best of our knowledge, the longest period validated planet found by TESS to date. The stellar brightness together with the expected Doppler semi-amplitude of $\sim 6 \mathrm{~m} \mathrm{~s}^{-1}$ make this target one of the few long-period transiting planets for which a precise mass measurement is feasible through radial velocity (RV) observations.

The host star is a subgiant that is in the process of moving away from the main-sequence and on to the red giant branch. Evolved stars are not normally prime targets for transit surveys, as their large radii make the transits shallower, longer, and harder to detect. They also display relatively large projected rotational velocities (owing to their large radii), making precise radial velocity measurements more difficult. Evolved stars are also comparatively scarce in the Solar neighbourhood, as the subgiant and giant phases of stellar evolution are short-lived. Consequently, relatively few planets are known around subgiants, yet these offer a unique opportunity to test how a mature planet responds to the increase in stellar flux and proximity as the star expands. This new discovery thus adds to the relatively small but important sample of known planets around evolved stars.

The layout of the remainder of this paper is as follows. We introduce the PHT project in Section 2, and describe the discovery of the TOI $813 \mathrm{~b}$ in the TESS data in Section 3. In Sections 4 and 5 , we report on the determination of the stellar parameters, and the statistical validation of the planet, respectively. The final planet parameters are derived and discussed in Section 6, and we present our conclusions in Section 7.

\section{THE CITIZEN SCIENCE APPROACH}

PHT is hosted by Zooniverse, the world's largest and most successful citizen science platform (Lintott et al. 2008, 2011). The primary

\footnotetext{
${ }^{1}$ https://exofop.ipac.caltech.edu/tess/

${ }^{2}$ www.planethunters.org
}

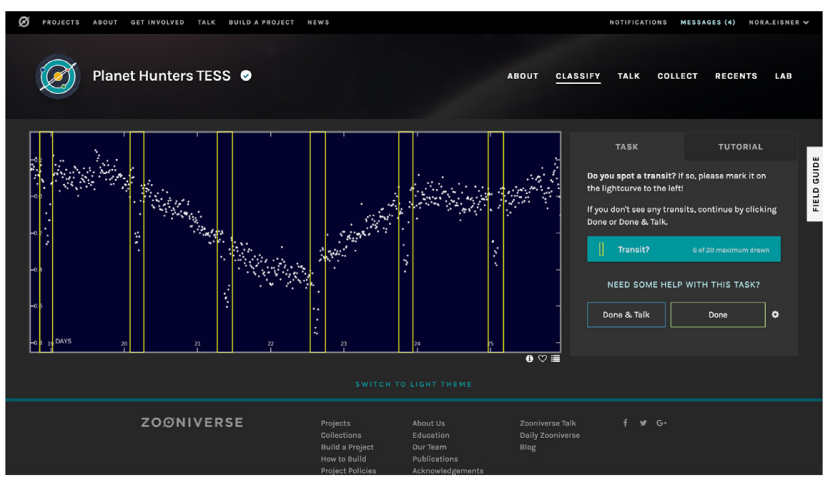

Figure 1. The PHT web interface, as it appeared for Sectors 1-10, with a randomly chosen $\sim 7 \mathrm{~d}$ light curve. Volunteers use a mouse drag to register transit-like events, as is shown by the yellow boxes.

goal of the project is to harness the power of citizen science to find transit events in the TESS data that were missed by the main TESS pipeline and by other teams of professional astronomers. The project works by displaying TESS light curves to volunteers and asking them to mark any transit-like signals by drawing a column over them, as shown in Fig. 1.

PHT builds on the success of the original Planet Hunters project (PH; Fischer et al. 2012), which used Kepler and K2 data. The initial $\mathrm{PH}$ had a detection efficiency $>85$ per cent for planets larger than $4 R_{\oplus}$ (Schwamb et al. 2012) and detected several noteworthy systems, including the first planet in a quadruple star system (Schwamb et al. 2013) and gas giants orbiting in the habitable zone of their host star (Wang et al. 2013; Schmitt et al. 2014a,b), as well as a large number of candidates that were not found by the main Kepler pipeline or other teams (Lintott et al. 2013; Wang et al. 2015). PH demonstrated that volunteers can outperform automated detection pipelines for certain types of transits, especially single (long-period) transits, as well as aperiodic transits (e.g. circumbinary planets; Schwamb et al. 2013) and planets around rapidly rotating, active stars (e.g. young systems; Fischer et al. 2012). Additionally, the highly unusual irregular variable KIC 8462852 was discovered as part of PH (Boyajian et al. 2016), highlighting the power of citizen science to identify rare but noteworthy objects.

Overall, the bulk of PH's contributions to the Kepler findings were long-period planet candidates. In fact, $\mathrm{PH}$ found $\sim 10$ per cent of the Kepler candidates with periods $>100$ d, and $\sim 50$ per cent of those with periods $>600 \mathrm{~d}$ (Fischer, private communication). This is because the $\mathrm{PH}$ volunteers typically identify transit-like events one by one, rather than by exploiting their periodic nature as most automated transit-search algorithms do. They are thus equally likely to find a planet candidate that produces only one transit event in a given light curve as they are to find multiple transit events, as was shown by Schwamb et al. (2012).

The importance of citizen science in the field of planet detection was also demonstrated by the highly successful Exoplanet Explorer (Christiansen et al. 2018) Zooniverse project, which used $K 2$ data. With the help of volunteers the project has so far led to the validation of multiple interesting planetary systems (e.g. Christiansen et al. 2018; Feinstein et al. 2019) as well as many new candidates (e.g. Zink et al. 2019).

The PHT project was launched with the first public TESS data release in 2018 December, and volunteers have since classified every 2-min cadence light curve from each Sector, typically within two weeks of that sector's release. By 2019 September 9, the PHT 
volunteers had classified almost $250000 \sim 30$ d light curves. Until Sector 10, the PHT interface was extremely similar to PH: each light curve was split into typically four $7 \mathrm{~d}$ segments, and pre-generated plots of the light curve, binned to 14-min sampling, were uploaded to the website and shown to the volunteers. As of Sector 11, the PHT interface displays the entire $\sim 27 \mathrm{~d}$ light curves binned to 10-min sampling, and the project has the added capability to zoom in on the data.

Volunteers are also shown simulated light curves where we have injected transit-like signals into light curves, resulting in an SNR of at least 7. These allow us to evaluate the sensitivity of the project and assess the skill of each individual volunteer. Each real light curve (or light-curve segment) is seen by 8 to 15 volunteers and the significance of each transit-like event is evaluated based on all the marked transits (a similar algorithm is described in Schwamb et al. 2012). Volunteers are also given the option to discuss their findings with each other, as well as with the science team, via the Talk discussion forum. ${ }^{3}$

PHT engages a very large number of members of the public, some of them over a considerable time period. There are $>11000$ registered participants, and many more who are not registered. Some spend only a few minutes on the site, others regularly devote several hours per week to the project. While most volunteers simply mark transit-like events, a significant proportion go much further, downloading and analysing TESS light curves at their own initiative.

\section{DISCOVERY OF TOI 813 B IN THE TESS DATA}

\subsection{TESS data}

TOI 813 (TIC 55525572; Stassun et al. 2019) is located at high ecliptic latitude and was observed almost continually by TESS during its first year of observations, from Sectors 1-13 except in Sector 7, according to the Web TESS Viewing Tool (WTV). ${ }^{4}$ However, it was only included in the list of targets for which 2-min cadence observations are available from Sector 4 onwards. Prior to that, i.e. during Sectors $1-3$, it was included in the Full Frame Images (FFIs) collected every 30 min only.

Only the 2-min cadence targets are searched by PHT, which uses the Pre-Search Data Conditioning (PDC-MAP) light curves produced by the TESS pipeline at the Science Processing Operations Center (SPOC; Jenkins et al. 2016). These light curves have been corrected for both known pixel-level instrumental effects and systematics common to many light curves. We downloaded the light curves from the Mikulski Archive for Space Telescopes (MAST) ${ }^{5}$ and discarded observations which were flagged by the SPOC pipeline as affected by various instrumental anomalies.

After the initial detection of the transits (see below), we used the FFIs for Sectors 1-3 to produce light curves for TOI 813 using the open source package eleanor (v0.1.8; Feinstein et al. 2019), which performs background subtraction, aperture photometry, and detrending for any source observed in the FFIs. The extracted FFI light curves were corrected for jitter by quadratically regressing with centroid position.

\footnotetext{
${ }^{3}$ http://www.zooniverse.org/projects/nora-dot-eisner/planet-hunters-TES S/talk

${ }^{4}$ https://heasarc.gsfc.nasa.gov/cgi-bin/tess/webtess/wtv.py

${ }^{5} \mathrm{http}: / /$ archive.stsci.edu/tess/
}

At the time of writing, the TESS data were publicly available up to, and including, Sector 13. The detailed analysis of TOI $813 \mathrm{~b}$ 's light curve, including the vetting checks described in Section 3.3 and the transit modelling (Section 6.1) was carried out using the 2-min cadence Light Curve (LC) files and Target Pixel Files (TPFs) produced by versions 3.3.51 to 3.3.75 of the SPOC pipeline.

In total, the light curve of TOI 813 consists of 149265 flux measurements between barycentric TESS Julian Date (BTJD, defined as BJD-2457000) 1354.13650 and 1682.35665 . The 2-min cadence light curves have a median flux of 18338 counts s$^{-1}$ and a typical RMS scatter of 122 counts $\mathrm{s}^{-1}$.

\subsection{Discovery of TOI $813 \mathrm{~b}$}

Adopting $~ 84-d$ as the orbital period of the companion, an additional transit was predicted to have taken place in Sector 2. The target was not included in PHT in Sector 2, as there was no 2-min cadence light curve. We thus extracted the Sector 2 light curve from the FFIs and found the transit by visual inspection at the expected time. A fourth transit was predicted to occur in Sector 11 data of the TESS primary Southern Hemisphere survey, and was indeed found in the Sector 11 light curve once that data were released. The full light curve for TOI 813 is shown in Fig. 2 and the individual transits in Fig. 3. Once the PHT team had completed basic vetting tests, we reported this candidate on the ExoFOP website ${ }^{6}$ as a community TESS Object of Interest (cTOI), and it was allocated TOI number 813.01.

At the time of the PHT discovery, 2019 April 27, TOI 813 was not listed as a TOI, nor did it have any threshold crossing events (TCEs). In other words, it was not detected by either the SPOC transit search pipeline, or the Quick Look Pipeline (Fausnaugh et al. 2018; Huang et al., in preparation), which is used by the TESS Science Office (TSO). This is because the planet never exhibits more than one transit in a given sector and the SPOC pipeline requires at least two transits for a detection. At the time, the SPOC pipeline had not yet been run on multisector data. The QLP light curve from Sector 8 did not advance past initial triage for vetting by the TOI team.

However, concurrently to the citizen science discovery, a different subset of the co-authors of this paper identified TOI $813 \mathrm{~b}$ independently, via a manual survey using the LCTOOLS software (Kipping et al. 2015), following the method described by Rappaport et al. (2019).

The TOI team released a TCE on TIC 55525572 as TIC 55525572.01 on 2019 June 21. The target appeared as a TCE in the SPOC multisector planet search in TESS sectors $1-9 .^{7}$ The target was initially ranked as low priority for manual vetting, and then classified as a potential planet candidate in group vetting. The first transit of the object occurred around the time of a spacecraft momentum dump, but the second was marked as a potential single transit of a planet candidate. Furthermore, the SPOC pipeline detected the transits of TOI-813 in sectors 5, 8, and 11 in the multisector transit search of sectors 1-13 performed on 2019 August 7 with orbital parameters and planet radius consistent with this work. ${ }^{8}$

\footnotetext{
${ }^{6}$ https://exofop.ipac.caltech.edu/tess/index.php

${ }^{7}$ https://archive.stsci.edu/missions/tess/doc/tess_drn/tess_multisector_01_0 9_drn15_v03.pdf

${ }^{8}$ https://mast.stsci.edu/api/v0.1/Download/file/?uri = mast:TESS/product/t ess2018206190142-s0001-s0013-0000000055525572-00226_dvr.pdf
} 


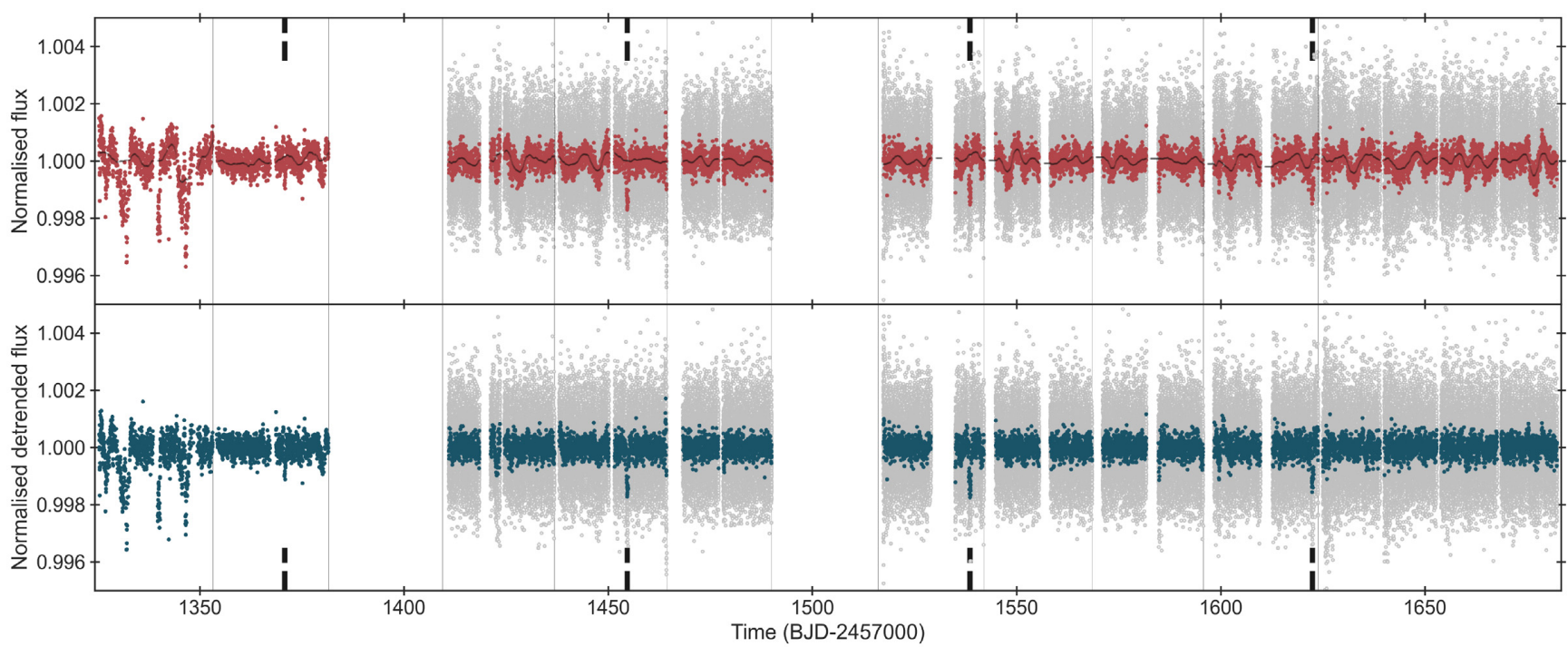

Figure 2. TESS light curve for TOI 813 for Sectors 1-13, except Sector 7. The top panel shows the PDC light curve at a 2-min sampling (grey) and a 30-min sampling (dark red). The black points show the best-fitting model used for detrending. The bottom panel shows the detrended light curve, used in the BLS search (see Section 3.4), also at a 2-min (grey) and 30-min (teal blue) sampling. The times of transits are indicated by the short black dashed vertical lines and the end of each sector is depicted by a solid light grey line.

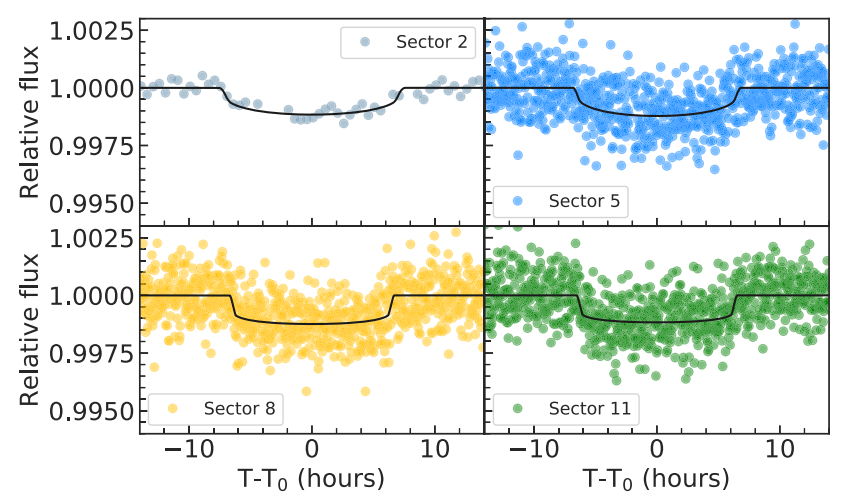

Figure 3. Individual transits of TOI $813 \mathrm{~b}$ in the TESS data in Sectors 2 (top left), 5 (top right), 8 (low left), and 11 (low right). The Sector 2 light curve was extracted from the FFIs whereas the other sectors are 2-min cadence observations.

\subsection{Light-curve-based vetting checks}

We carried out a number of vetting tests on the TESS data, similar to the 'Data Validation' step of the SPOC pipeline. These are intended to rule out as many as possible of the false positive scenarios that could have given rise to the detection, whether they are of instrumental or astrophysical origin.

First, we checked for instrumental false alarms by comparing the light curve around the time of each transit to the star's centroid position and the background flux, which are provided in the lightcurve files, and to the times of reaction wheel momentum dumps, which occur every 2 to $2.5 \mathrm{~d}$ and typically last around half an hour. While observations taken during a momentum dump are flagged and were not used in this work, the satellite pointing remains affected for several hours after each dump, and can result in spurious flux variations due to aperture losses or inter-/intrapixel sensitivity variations. Additionally, enhanced scattered light in the telescope optics can cause dramatic increases in the background flux when the Earth, Moon, or other Solar system planet pass below $25^{\circ}$ from the boresight of any of the cameras. Both of these effects can induce spurious transit-like events. Even though one of the transits of TOI $813 \mathrm{~b}$ was found to occur at the time of a momentum dump, the other three do not coincide with times where the light curve was potentially affected by enhanced pointing jitter or background flux, and due to the periodicity of all of the transit-events, we believe them all to be real.

In order to identify other sections of the light curves potentially affected by residual systematics common to the light curves of many different targets, we plotted a histogram of all of the volunteer markings across all subject light curves for each sector. If volunteers tend to mark transit-like events at the same time across many sources, it is likely that these are caused by systematics. We found that none of the transits of TOI $813 \mathrm{~b}$ observed in Sectors 5, 8, or 11 coincide with a time when volunteers marked an unusually high number of other targets. Finally, we inspected the light curves of all TESS 2-min target stars within $0.5^{\circ}$ of TOI 813 in order to check whether any of them showed flux dips at the times of the transits, finding none. This allowed us to rule out large-scale detector anomalies or contamination by a bright, nearby eclipsing binary as the cause for the transit-like signals.

We then carried out a further set of vetting tests aiming to exclude astrophysical false positives. In particular, TESS's large pixel size (21 arcsec) means that many fainter stars contribute to the flux recorded in the aperture of each target. If any of these faint neighbour is an eclipsing binary, its diluted eclipses can mimic a transit on the main target star. A large fraction of these 'blend' scenarios can be ruled out as follows:

Odd and even transit comparison: We compared the depths, duration, and shape of the odd- and even-numbered transits. Slight differences in these would indicate that the 'transits' are caused by a near-equal mass eclipsing binary. The phase-folded light curves for the odd- and even-numbered transits were modelled separately (as described in Section 6.1) and were found to have depths and widths consistent with one another to within $0.5 \sigma$. 

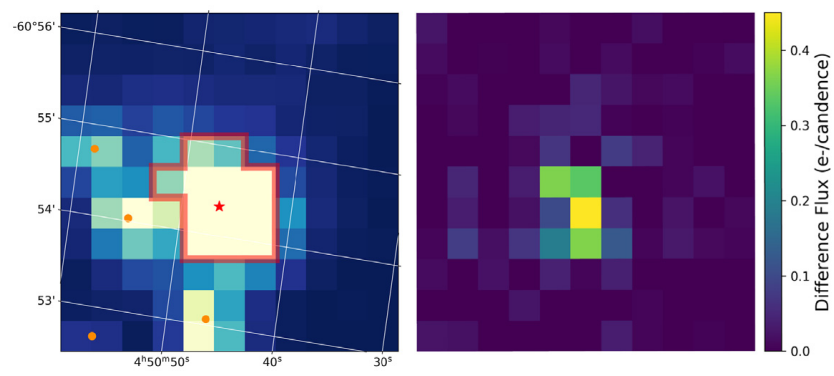

Figure 4. Left-hand panel: TESS image in the vicinity of TOI 813 throughout Sector 5. The red outline shows aperture mask used to extract the light curve. Neighbouring stars brighter than $V=16$ within $180 \operatorname{arcmin}$ of TOI 813 are depicted by orange dots. Right-hand panel: Difference in/out of transit image during Sector 5 .

Secondary eclipse search: We searched for a secondary eclipse (or occultation), which might indicate that the transits are caused by a small but self-luminous companion, such as a brown dwarf or low-mass star. This was done by conducting a grid search for the deepest out-of-transit signal in the phase-folded light curve (see e.g. Rowe et al. 2015). This resulted in an upper limit on the depth of any secondary eclipse at the level of 346 parts per million (ppm).

Pixel-level centroid analysis: A blend with a background eclipsing binary would result in a change in the spatial distribution of the flux within the target aperture during the transits. We checked for this in two ways. First, we computed a difference image by subtracting the in-transit pixel flux from the image obtained during a similar time period immediately before and after each transit. This was done using the Target Pixel Files (TPFs) released alongside the 2-min cadence light curves, after correcting them for systematic effects using Principal Component Analysis (e.g. Smith et al. 2012; Stumpe et al. 2012). The difference image shows only one source, whose location coincides with that of the main target, as shown for the Sector 5 transit in the right-hand panel of Fig. 4. Secondly, we compared the observed position of the target during, and immediately before and after each transit but no significant differences were found. To quantify this, we used a two-sided Kolmogorov-Smirnov test to see whether the fluxweighted centroid positions in- and out-of-transit are drawn from the same distribution or not. This non-parametric test showed that for all of the transits the detrended $\mathrm{x}$ - and $\mathrm{y}$-centroid positions did not differ significantly in-transit compared to out-of-transit, with $p$-values ranging from 0.34 to 0.98 (where statistically different is defined as $p<0.05)$.

Light-curve extraction with different aperture sizes: Another signature of a blended eclipsing binary would be a change in the depth of the transit depending on the size of the photometric aperture used. To check for this, we extracted the 2-min cadence light curve with different photometric apertures (by growing or shrinking the default aperture mask shown in the left-hand panel of Fig. 4 by one pixel). For the 30-min cadence data, we extracted the light curves with different aperture sizes using ELEANOR. This analysis, which was carried out for each transit event independently, showed that the aperture size had a minimal effect on the depth and shape of the transit.

Nearby companion stars: We searched for evidence of nearby stars by querying all entries in the Gaia Data Release 2 catalogue (Gaia Collaboration 2018) within 180 arcsec of TOI 813. We found there to be four stars within this radius with magnitudes brighter than $V=16$, as shown in Fig. 4). The SPOC pipeline's difference imaging analysis, however, showed that none of these stars are located within the 3-sigma confusion region around the target. Nonetheless, in order to rule out the closest companion stars we calculated the magnitude difference between TOI 813 and the faintest companion star that could plausibly cause the observed a transit depth and shape. We used the equations presented in Vanderburg et al. (2019) and the transit parameters derived using a Markov Chain Monte Carlo (MCMC) approach (see Section 6.1) and found the maximum magnitude difference to be $0.4 \mathrm{mag}$. This allows us to confidently rule out the 14.636 magnitude star located at an angular separation of $\sim 19 \operatorname{arcsec}$ as the cause of the transit signal. Furthermore, the SPOC pipeline (Jenkins et al. 2016) accounts for the contamination of the aperture by the neighbouring stars, so that we do not need to correct the measured transit depth for the effect of this light.

The above tests rule out many, but not all, of the plausible astrophysical false positive scenarios. While we can state with confidence that none of the nearby stars brighter than $V=16$ (shown in Fig. 4) are the source of the transits, fainter contaminants located nearer the main target cannot be ruled out at this stage. Nonetheless, these vetting tests increased our confidence in the planetary nature of the companion sufficiently to motivate ground-based follow-up observations.

We also note that TOI $813 \mathrm{~b}$ independently passed all of the SPOC pipeline Data Validation diagnostic tests in the multisector 1-13 run. These tests included the odd and even transit comparison test, the weak secondary eclipse search test, the ghost diagnostic test (which is similar to this work's increasing aperture size test), and the statistical bootstrap test, which looks at the propensity of other transit-like features in the light curve to cause happenstance false positives (Twicken et al. 2018; Li et al. 2019).

\subsection{Search for additional planets}

The discovery of multiple planets within one system can significantly increase confidence in the planetary nature of a transit-like event. No additional planet candidates were identified by the citizen science campaign, however, the volunteers' detection efficiency is known to decrease notably as a function of planet radius, as was shown by the initial PH project (Schwamb et al. 2012). We therefore carried out a search for additional transits in the full light curve using the Box Least Squares (BLS; Kovács, Zucker \& Mazeh 2002) algorithm, after masking the transits of TOI $813 \mathrm{~b}$. Before running the BLS, we used an iterative non-linear filter (Aigrain \& Irwin 2004) to estimate and subtract residual systematics on time-scales $>1.7 \mathrm{~d}$ (see Fig. 2). The BLS search was carried out on an evenly sampled frequency grid ranging from 0.01 to $1 \mathrm{~d}^{-1}$ ( 1 to $90 \mathrm{~d}$ ). We used the ratio of the highest peak in the SNR periodogram relative to its standard deviation, known as the signal detection efficiency (SDE) to quantify the significance of the detection. The algorithm found no additional signals with SDE > 7.2 (compared to SDE 22.4 for the transit of TOI $813 \mathrm{~b}$ ).

In addition, we searched for further companions by manually inspecting the light curve with LcTools, after having binned the data at 6 points per hour in order to visually enhance undetected signals with low SNRs. No additional transits were identified with either method.

The non-detection of additional transits suggests that if there are other planets in orbits interior to TOI $813 \mathrm{~b}$, their orbits are either inclined to not transit or are shallower than the TESS detection threshold. 


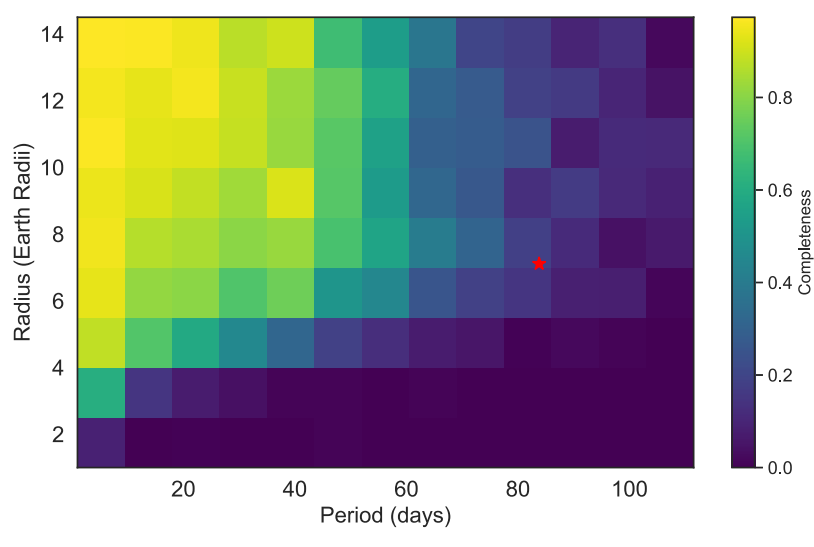

Figure 5. The recovery completeness of injected transit signals into the light curve of TOI 813 as a function of the radius versus orbital period, where the signals were recovered using a BLS search. The properties of TOI $813 \mathrm{~b}$ are depicted by the red star.

We used injection and recovery tests to quantify the detectability of additional planets in the TESS light curve of TOI 813, injecting artificial signals into the PDC light curve before repeating the masking of TOI 813 b's transit, the detrending and the BLS search. The injected transits were generated using the open source batman package (Kreidberg 2015), with planet radii and orbital periods sampled at random from logarithmic distributions ranging from 1 to $7.11 R_{\oplus}$ and from 0 to $120 \mathrm{~d}$, respectively. The upper bound of the planet radius distribution was chosen to coincide with our best-fitting radius for TOI 813 b. For simplicity, the impact parameter and eccentricity were assumed to be zero throughout. We used a quadratic limbdarkening law with $q_{1}$ and $q_{2}$ of 0.59 and 0.22 , respectively (See Table 3).

We simulated and injected transits for 50000 planets and attempted to detect them using the BLS algorithm as was done when searching for real signals. For each simulation we identified the highest peak in the BLS periodogram and recorded the corresponding period and orbital phase. The injected signal was considered to be correctly identified if the recovered period and orbital phase were within 1 per cent of the injected values. We then evaluated the completeness of our search for additional transits by computing the fraction of injected transits that were correctly identified over a grid of period and radius. As we injected the simulated transits into the PDC light curve, the derived detection limits do not take into account the impact of the PDC-MAP systematics correction on transit events, and should therefore be considered optimistic.

The results are shown in Fig. 5. We recover more than 80 per cent of the simulated planets larger than $\sim 5 R_{\odot}$ with periods less than $30 \mathrm{~d}$, dropping to 50 percent for periods less than $60 \mathrm{~d}$. Therefore, we cannot rule out the presence of additional planets transiting inside the orbit of TOI $813 \mathrm{~b}$ at high confidence, particularly sub-Neptunes. It is also interesting to note that the completeness for the period and radius of TOI $813 \mathrm{~b}$ itself is rather low ( $\sim 30$ per cent): even with multiple transits, such long-period planets are relatively hard to detect in TESS data using standard algorithms. Nonetheless, these single transit events are often visible by the human eye, thus highlighting the importance of citizen science.

\section{REFINING THE STELLAR PARAMETERS}

The stellar parameters provided in the TIC (Stassun et al. 2019) are based on broad-band photometry, and their precision is therefore limited. We thus acquired moderate, then high-dispersion spectra of TOI 813 to refine our estimate of the host star's parameters.

\subsection{Spectroscopy}

We first obtained a moderate spectral resolution $(R=7000)$ spectrum of TOI 813 with the Wide Field Spectrograph instrument on the Australian National University (ANU) 2.3-m telescope (Dopita et al. 2007) on the night of 2019 April 30. We obtained three 120 s exposures with the U7000 (SNR 400) and R7000 (SNR $\sim 700$ ) gratings and the data were reduced using the pyWiFeS data reduction pipeline version 0.7.4 (Childress et al. 2014).

We then acquired a high-resolution $(R \approx 115000)$ spectrum with the High Accuracy Radial velocity Planet Searcher (HARPS; Mayor et al. 2003) spectrograph on the ESO 3.6-m telescope at La Silla observatory (Chile). The observations were carried out on 2019 July 14 as part of observing program 1102.C-0923. We used an exposure time of 1800 s leading to an SNR per pixel of $\sim 60$ at $5500 \AA$.

The spectrum was reduced and extracted using the standard HARPS Data Reduction Software (DRS; Baranne et al. 1996). This much higher resolution spectrum displayed no obvious signs of binarity, and was used to obtain the final estimate of the stellar parameters, using the method described (Section 4.2) below.

\subsection{Stellar parameters}

We used the python package ispec (Blanco-Cuaresma et al. $2014)$ to derive the stellar effective temperature, $T_{\text {eff }}$, as well as the surface gravity, $\log g_{\star}$, and stellar metallicity, $[\mathrm{Fe} / \mathrm{H}]$ from the ANU spectrum. We then derived the atmospheric parameters by comparing our data with synthetic spectra. Our modelling used the code spectrum (Gray \& Corbally 1994), with atmospheric models taken from ATLAS9 and the atomic line list from the VALD data base. ${ }^{9}$ (Ryabchikova et al. 2015) The ispecanalysis produced $T_{\text {eff }}=5700 \pm 120 \mathrm{~K},[\mathrm{~F} / \mathrm{H}]=0.05 \pm 0.10$, and $\log g_{\star}=3.85 \pm 0.04$ ( $\left.\mathrm{gcc}\right)$.

We also used the Spectroscopy Made Easy (sme; Valenti \& Piskunov 1996; Piskunov \& Valenti 2017) code to estimate the stellar parameters from the HARPS spectrum. sme works by calculating the synthetic stellar spectra from grids of detailed atmosphere models and fitting them to the observations with a chi-square-minimization approach. We used sme Version 5.22 with the ATLAS12 model spectra (Kurucz 2013) to derive $T_{\text {eff }}$, $\log g_{\star},[\mathrm{Fe} / \mathrm{H}]$, and the $v \sin i_{\star}$. All of these parameters were allowed to vary throughout the model fitting, while the microand macroturbulence ( $v_{\text {mic }}$ and $v_{\text {mac }}$ ) were fixed through empirical calibration equations (Bruntt et al. 2010; Doyle et al. 2014) valid for Sun-like stars after a first estimation of $T_{\text {eff. }}$. The required atomic and molecular parameters were taken from the vald data base (Ryabchikova et al. 2015). A detailed description of the methodology can be found in Fridlund et al. (2017) and Persson et al. (2018). We derived $T_{\text {eff }}=5907 \pm 150 \mathrm{~K}, \log g_{\star}=3.86 \pm 0.14$ (gcc) from Mg I lines, $\log g_{\star}=3.85 \pm 0.20$ (gcc) from Ca I lines, $[\mathrm{Fe} / \mathrm{H}]=0.10 \pm 0.10$ dex and $v \sin i_{\star}=8.2 \pm 0.9 \mathrm{~km} \mathrm{~s}^{-1}$.

\footnotetext{
${ }^{9} \mathrm{http}: / /$ vald.astro.uu.se
} 
Table 1. Stellar parameters of TOI 813.

\begin{tabular}{|c|c|c|}
\hline Parameter & Value & Source \\
\hline \multicolumn{3}{|l|}{ Identifiers } \\
\hline TIC & 55525572 & Stassun et al. (2019) \\
\hline Gaia DR2 & 4665704096987467776 & Gaia DR2 ${ }^{\mathrm{a}}$ \\
\hline 2MASS & J04504658-6054196 & $2 \mathrm{MASS}^{\mathrm{b}}$ \\
\hline \multicolumn{3}{|l|}{ Astrometry } \\
\hline$\alpha_{\mathrm{J} 2000}$ & $04: 50: 46.57$ & Gaia DR2 $2^{a}$ \\
\hline$\delta \mathrm{J} 2000$ & $-60: 54: 19.62$ & Gaia DR2 ${ }^{a}$ \\
\hline Distance (pc) & $265.1535 \pm 1.582$ & Gaia DR2 ${ }^{a}$ \\
\hline$\pi$ (mas) & $3.7714 \pm 0.0225$ & Gaia DR2 $^{a}$ \\
\hline \multicolumn{3}{|l|}{ Photometry } \\
\hline$B_{T}$ & $11.189 \pm 0.056$ & Tycho- $2^{c}$ \\
\hline$V_{T}$ & $10.435 \pm 0.056$ & Tycho- $2^{c}$ \\
\hline$B$ & $10.905 \pm 0.026$ & $\mathrm{APASS}^{d}$ \\
\hline$V$ & $10.322 \pm 0.014$ & $\mathrm{APASS}^{d}$ \\
\hline$g$ & $10.587 \pm 0.015$ & $\mathrm{APASS}^{d}$ \\
\hline$r$ & $10.240 \pm 0.054$ & $\mathrm{APASS}^{d}$ \\
\hline$i$ & $10.094 \pm 0.044$ & $\operatorname{APASS}^{d}$ \\
\hline$G$ & $10.2352 \pm 0.0004$ & Gaia DR2 $^{a}$ \\
\hline$J$ & $9.326 \pm 0.021$ & $2 \mathrm{MASS}^{b}$ \\
\hline$H$ & $9.053 \pm 0.018$ & $2 \mathrm{MASS}^{\mathrm{b}}$ \\
\hline$K_{s}$ & $9.029 \pm 0.019$ & $2 \mathrm{MASS}^{\mathrm{b}}$ \\
\hline$W 1(3.35 \mu \mathrm{m})$ & $8.992 \pm 0.023$ & WISE $^{e}$ \\
\hline$W 1(4.6 \mu \mathrm{m})$ & $9.027 \pm 0.020$ & WISE $^{e}$ \\
\hline$W 1(11.6 \mu \mathrm{m})$ & $8.971 \pm 0.022$ & WISE $^{e}$ \\
\hline$W 1(22.1 \mu \mathrm{m})$ & $9.126 \pm 0.271$ & WISE $^{e}$ \\
\hline \multicolumn{3}{|l|}{ Kinematics } \\
\hline \multicolumn{3}{|l|}{ Physical properties } \\
\hline Stellar mass $M_{\star}\left(M_{\odot}\right)$ & $1.32 \pm 0.06$ & This work \\
\hline Stellar radius $R_{\star}\left(R_{\odot}\right)$ & $1.94 \pm 0.10$ & This work \\
\hline$v \sin i_{\star}\left(\mathrm{km} \mathrm{s}^{-1}\right)$ & $8.2 \pm 0.9$ & This work \\
\hline Stellar density $\rho_{\star}\left(\mathrm{g} \mathrm{cm}^{-3}\right)$ & $0.254_{-0.037}^{+0.046}$ & This work \\
\hline Effective temperature $\mathrm{T}_{\mathrm{eff}}(\mathrm{K})$ & $5907 \pm 150$ & This work \\
\hline Surface gravity $\log g_{\star}$ from $\mathrm{Mg} \mathrm{I}(\mathrm{gcc})$ & $3.86 \pm 0.14$ & This work \\
\hline Surface gravity $\log g_{\star}$ from Ca I ( $\left.\mathrm{gcc}\right)$ & $3.85 \pm 0.20$ & This work \\
\hline Iron abundance $[\mathrm{Fe} / \mathrm{H}](\mathrm{dex})$ & $0.10 \pm 0.10$ & This work \\
\hline Star age (Gyr) & $3.73 \pm 0.62$ & This work \\
\hline Spectral Type & G0 IV & Pecaut \& Mamajek (2013) \\
\hline$v_{\text {mic }}\left(\mathrm{km} \mathrm{s}^{-1}\right)$ & 4.4 & Doyle et al. (2014) \\
\hline$v_{\text {mac }}\left(\mathrm{km} \mathrm{s}^{-1}\right)$ & 1.21 & Bruntt et al. (2010) \\
\hline
\end{tabular}

Note. ${ }^{\mathrm{a}}$ Gaia Data Release 2 (DR2; Gaia Collaboration 2018).

${ }^{b}$ Two-micron All Sky Survey (2MASS; Skrutskie et al. 2006).

${ }^{\mathrm{c}}$ Tycho-2 catalogue (Høg et al. 2000).

${ }^{d}$ AAVSO Photometric All-Sky Survey (APASS; Munari et al. 2014).

${ }^{e}$ Wide-field Infrared Survey Explorer catalogue (WISE; Wright et al. 2010)

The HARPS spectrum was independently analysed using the specmatch-emp package (Yee, Petigura \& von Braun 2017), which compares the observed spectrum with a library of $\approx 400$ synthetic spectra of FGK and $\mathrm{M}$ stars. This fitting routine also uses a chisquared minimization approach to derive the stellar parameters, and yielded the values $T_{\text {eff }}=6006 \pm 110 \mathrm{~K},[\mathrm{Fe} / \mathrm{H}]=0.17 \pm 0.09 \mathrm{dex}$, $R=1.756 R_{\odot}$. These values are consistent with those found in the SME analysis, but the latter have slightly larger error bars, which we consider more realistic. We therefore adopted the values derived with SME, which are reported in Table 1, for all further analysis.
We derived the stellar mass, radius, and age using the on-line interface param-1. $3^{10}$ with parsec stellar tracks and isochrones (Bressan et al. 2012), the Gaia parallax ( $\pi=3.7714 \pm 0.0225$ mas; Gaia Collaboration 2018), a $V$-band magnitude of 10.286 (Munari et al. 2014) and the stellar parameters derived from the sme analysis of the HARPS data. Munari et al. (2014) reported an interstellar reddening consistent with zero, so we did not correct the $V$ mag reported in Table 1.

As an independent check on the derived stellar parameters, we performed an analysis of the broad-band spectral energy distribution 


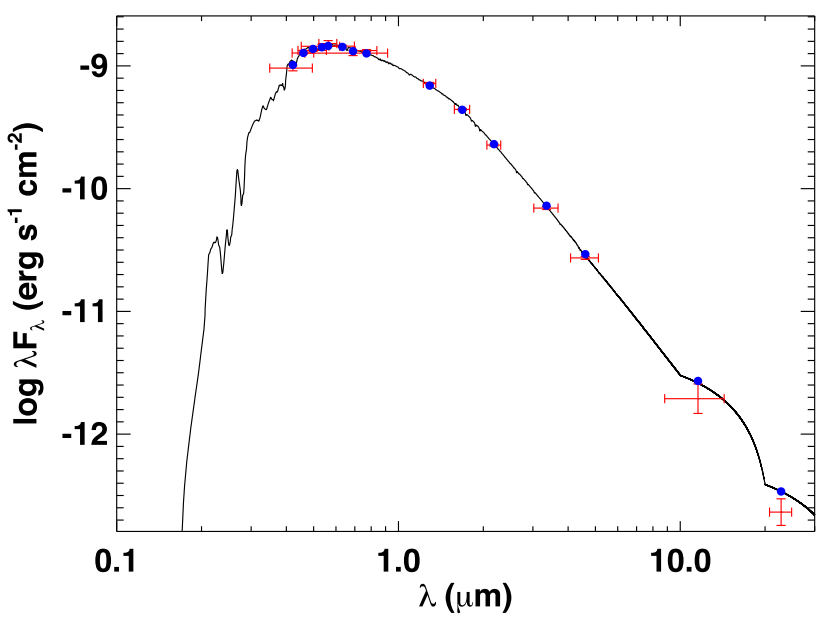

Figure 6. Spectral energy distribution (SED) of TOI 813. The red symbols represent the observed photometric measurements, where the horizontal bars represent the effective width of the passband. The blue symbols are the model fluxes from the best-fitting Kurucz atmosphere model (black).

(SED) together with the Gaia DR2 parallax in order to determine an empirical measurement of the stellar radius, following the procedures described in Stassun \& Torres (2016), Stassun, Collins \& Gaudi (2017), and Stassun et al. (2018). We retrieved the $B_{T} V_{T}$ magnitudes from Tycho-2, the BVgri magnitudes from APASS, the $J H K_{S}$ magnitudes from 2MASS, the W1-W4 magnitudes from WISE, and the $G$ magnitude from Gaia (see Table 1). Together, the available photometry spans the full stellar SED over the wavelength range $0.4-22 \mu \mathrm{m}$ (see Fig. 6). We performed a fit using Kurucz stellar atmosphere models, with the priors on effective temperature $\left(T_{\text {eff }}\right)$, surface gravity $(\log g)$, and metallicity $([\mathrm{Fe} / \mathrm{H}])$ from the spectroscopically determined values. The remaining free parameter is the extinction $\left(A_{V}\right)$, which we restricted to the maximum lineof-sight value from the dust maps of Schlegel, Finkbeiner \& Davis (1998). The resulting fits are excellent (Fig. 6) with a reduced $\chi^{2}$ of 2.5. The best-fitting extinction is $A_{V}=0.00_{-0.00}^{+0.01}$. Integrating the (unreddened) model SED gives the bolometric flux at Earth of $F_{\mathrm{bol}}=$ $1.852 \pm 0.021 \times 10^{-9} \mathrm{erg} \mathrm{s} \mathrm{cm}^{-2}$. Taking the $F_{\text {bol }}$ and $T_{\text {eff }}$ together with the Gaia DR2 parallax, adjusted by +0.08 mas to account for the systematic offset reported by Stassun \& Torres (2018), gives the stellar radius as $R=1.891 \pm 0.097 \mathrm{R}_{\odot}$. Finally, estimating the stellar mass from the empirical relations of Torres, Andersen \& Giménez (2010a) gives $M=1.39 \pm 0.10 \mathrm{M}_{\odot}$, which with the radius gives the mean stellar density $\rho=0.290 \pm 0.049 \mathrm{~g} \mathrm{~cm}^{-3}$. These agree well with the spectroscopically derived parameters.

As a subgiant, TOI 813 is expected to display solar-like (pmode) oscillations which, if detected, could provide an independent estimate of the stellar parameters. We performed a search for such oscillations using the Lomb-Scarge periodogram (Lomb 1976; Scargle 1982), but did not detect any evidence of oscillations. The TESS Asteroseismology Consortium (TASC) was also unable to detect oscillations in this object (W. Chaplin, private communication).

\section{PLANET VALIDATION}

\subsection{High resolution imaging}

We performed speckle imaging using the Zorro instrument on the 8.1-m Gemini South telescope (Matson, Howell \& Ciardi 2019)
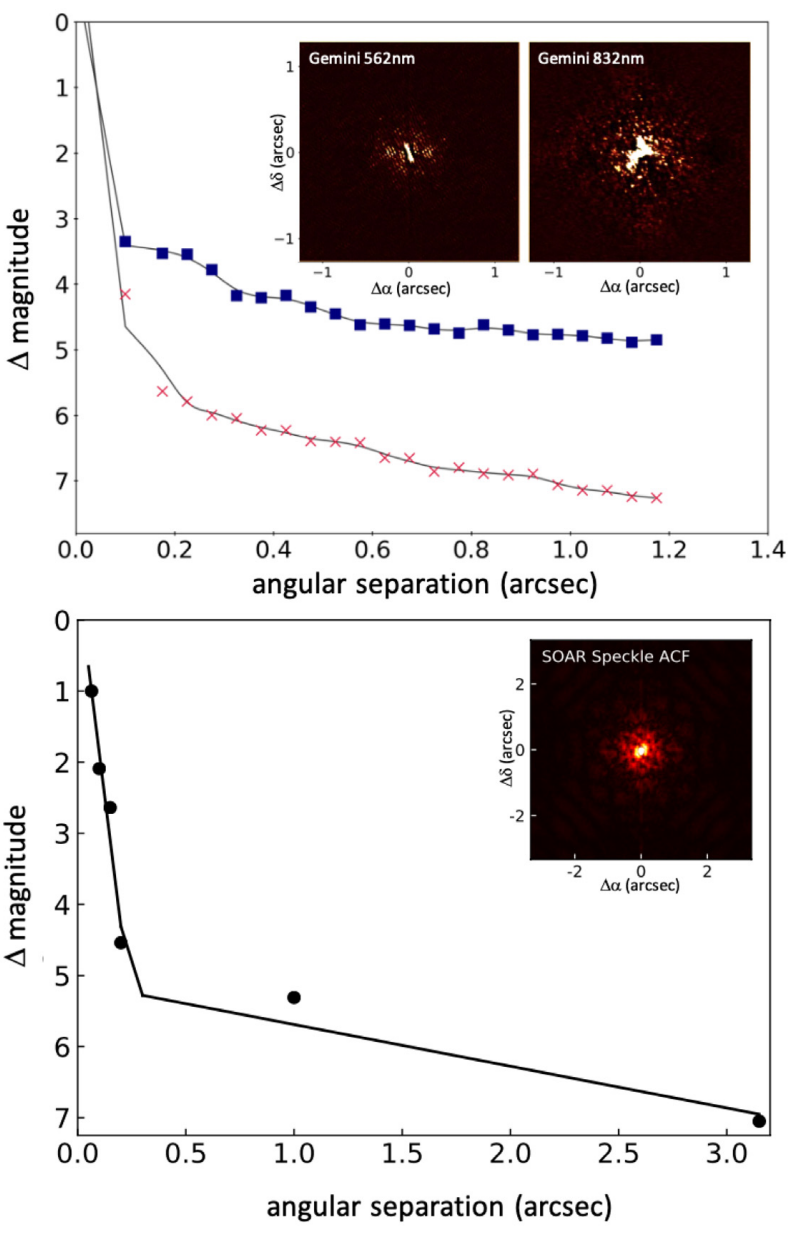

Figure 7. Contrast curves showing the $5 \sigma$ detection sensitivity and speckle autocorrelation functions. The top panel shows the data obtained using Zorro on Gemini with the filters centred on $562 \mathrm{~nm}$ (blue squares) and $832 \mathrm{~nm}$ (red crosses) and the bottom panel shows the observations obtained with the HRCam speckle imager on SOAR in the $I$-filter.

in order to search for close companions and to quantify their contribution to the TESS photometric aperture. Observations were obtained on the night of 2019 July 16 using simultaneous twocolour diffraction-limited optical imaging with $60 \mathrm{msec}$ exposures in sets of 1000 frames. The fast exposure times and rapid read out effectively 'freeze out' atmospheric turbulence. The image was reconstructed in Fourier space, a standard method for speckle image processing (Howell et al. 2011). We detect no companions within $1.17 \mathrm{arcsec}$ of the target at the 4-5 $\Delta$ mag limit at $562 \mathrm{~nm}$ and 5-7 $\Delta$ mag limit at $832 \mathrm{~nm}$.

Additionally, we searched for nearby sources to TOI 813 with speckle images obtained using the HRCam speckle imager on the 4.1-m Southern Astrophysical Research (SOAR; Tokovinin 2018) telescope at Cerro Pachon Observatory. The I-band observations, obtained as part of the SOAR TESS Survey (Ziegler et al. 2020) on the night of the 2019 July 14, show no evidence of any faint companions within 3 arcsec of TOI 813 up to a magnitude difference of $7 \mathrm{mag}$.

The $5 \sigma$ detection sensitivity and the speckle autocorrelation function from the SOAR and Gemini observation, as well as the speckle autocorrelation functions, are plotted in Fig. 7. 
Table 2. Relative radial velocity measurements of TOI 813 .

\begin{tabular}{lc}
\hline $\mathrm{RV}\left(\mathrm{m} \mathrm{s}^{-1}\right)$ & Uncertainties $\left(\mathrm{m} \mathrm{s}^{-1}\right)$ \\
\hline 12.6 & 27.2 \\
21.7 & 30.6 \\
18.7 & 27.9 \\
\hline
\end{tabular}

\subsection{Radial velocity monitoring}

We collected a total of four spectra of TOI 813 using the CHIRON high-resolution echelle spectrograph (Tokovinin et al. 2013) between 2019 September 13 and October 26, in order to confirm that the mass of TOI $813 \mathrm{~b}$ is consistent with that of a planetary body $\left(M<13 M_{\text {Jup }}\right)$. The CHIRON instrument, which is mounted on the CTIO/SMARTS 1.5-m telescope at the Cerro Tololo Inter-American observatory in Chile, has a 2.7 arcsec diameter multimode optical fibre and we used the high efficient image slicer mode in order to obtain a spectral resolution of $R \sim 80000$. The median SNR across the four observations was found to be around 15-20 for the median order. The observations were wavelength calibrated by the CHIRON pipeline and corrected for night drift using Th-Ar spectra, which were taken before and after each science exposure. The correction for the motion of the Earth about the barycentre of the Solar system was calculated using Barycorrpy (Kanodia \& Wright 2018). The 59 individual orders were normalized by fitting each one with a sinesquared blaze function plus a low-order polynomial to represent the stellar continuum, using iterative $k$-sigma clipping to exclude strong absorption lines from the fit.

We extracted relative radial velocity (RV) measurements from the spectra using a novel, template-free approach based on Gaussian Processes (GP) regression. Specifically, all available spectra were fit using a single GP model (using a Matern 3/2 kernel), allowing for small shifts in RV between the different epochs. This approach makes no assumptions about the stellar spectrum, except that it is not intrinsically variable. The number of free parameters of the fit were thus $N+1$, where $N=4$ is the number of epochs: $N-1 \mathrm{RV}$ shifts and 2 GP covariance parameters (input and output scales). After performing a maximum-likelihood fit to obtain an initial estimate of the hyperparameters, we used the EMCEE MCMC package (ForemanMackey et al. 2013) to compute median RV estimates and their $1-\sigma$ confidence intervals (using improper uniform priors for the RV shifts, and log-uniform priors for the GP covariance parameters). The RV extraction was performed independently for each of the 59 echelle orders. At this stage we identified and excluded 30 orders which systematically gave highly discrepant RVs (more than five standard deviations away from the median of the other orders), before combining the remaining RV estimates into a final value for each epoch using inverse variance weighting. The resulting three relative RV measurements and their uncertainties are listed in Table 2 .

The RV measurements were then used to constrain the maximum RV amplitude induced by the transiting companion. This was done by fitting for a circular orbit using the ephemeris found in the transit analysis (see Section 6.1). The open source code pyaneti (Barragán, Gandolfi \& Antoniciello 2019) was used to sample for an instrumental offset and the induced Doppler semiamplitude, adopting a uniform prior on the semi-amplitude between 0 and $1 \mathrm{~km} \mathrm{~s}^{-1}$. Fig. 8 shows the CHIRON data together with the inferred model. We obtained a median value of the semi-amplitude

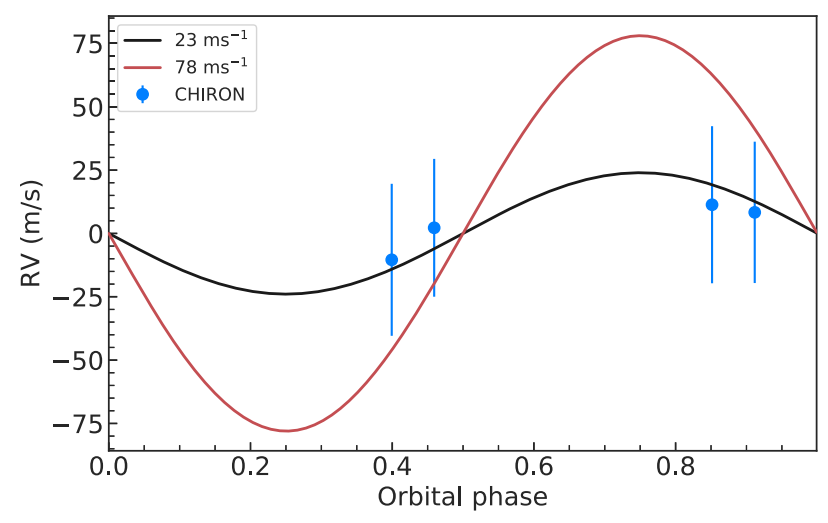

Figure 8. CHIRON RV data (blue points with error bars). A model with semi-amplitude of $23 \mathrm{~m} \mathrm{~s}^{-1}$ (corresponding to the median of the credible interval) is shown with a black line. A model with semi-amplitude of $78 \mathrm{~m} \mathrm{~s}^{-1}$ (corresponding to the 99 per cent credible interval) is shown with a red line.

of $23 \mathrm{~m} \mathrm{~s}^{-1}$ with a limit (at the 99 percent credible interval) of $78 \mathrm{~m} \mathrm{~s}^{-1}$, corresponding to a maximum planet mass of $2 M_{\text {Jup }}$. This is consistent with a planet scenario. We note that this is a preliminary analysis and that a more detailed approach would likely yield more precise RV measurements from the same data. Nonetheless, the constraints that we are able to place using this method are efficient to confirm the planetary nature of TOI $813 \mathrm{~b}$.

\subsection{Statistical validation}

The most direct way to confirm the planetary nature of TOI $813 \mathrm{~b}$ would be to measure the RV wobble it induces in its host star, and thereby measure the companion's mass. However, for such a longperiod object this will take at least a full season of RV observations, and is beyond the scope of this paper. On the other hand, we are in a position to validate the planetary nature of TOI $813 \mathrm{~b}$ in the statistical sense, by evaluating the likelihood that the transit signals are caused by a planet as opposed to the range of alternative, astrophysical false positive scenarios, using all of the available data. Statistical validation of transiting planets became routine in the era of Kepler (e.g. Borucki et al. 2012; Morton 2012; Díaz et al. 2014; Santerne et al. 2015), as many of the systems detected by that satellite are too faint for high-precision RV follow-up, and can be applied in much the same way to TESS targets.

We used the open source package vespa (Morton 2012, 2015), which calculates the false positive probability (FPP) by considering three astrophysical false positive scenarios: undiluted eclipsing binary (EB); eclipsing binary that is diluted by another star, often known as a background eclipsing binary (BEB); and hierarchical triple eclipsing binary (HEB). We used version 0.4 .7 of the software (with the MultiNest backend) with the following inputs: the 2min cadence detrended phase folded TESS light curve, the stellar parameters as derived in Section 4.2 and listed in Table 1, the two contrast curves derived from the SOAR and Gemini speckle images (Section 5.1), and the upper limit of the depth of a potential secondary eclipse (Section 3.3). We found the FPP to be 0.003 , which is below the commonly used validation threshold of FPP $<$ 0.01 (e.g. Montet et al. 2015; Morton et al. 2016; Livingston et al. 2018), allowing us to conclude TOI 813 b is a non-self-luminous object transiting the main target star. 
Table 3. System parameters.

\begin{tabular}{|c|c|c|}
\hline Parameter & Prior $^{a}$ & Value $^{b}$ \\
\hline \multicolumn{3}{|l|}{ Model Parameters for TOI $813 b$} \\
\hline Orbital period $P_{\text {orb }}(\mathrm{d})$ & $\mathcal{U}[83.7945,83.9859]$ & $83.8911_{-0.0031}^{+0.0027}$ \\
\hline Transit epoch $T_{0}$ (BJD - 2457000$)$ & $\mathcal{U}[1454.5781,1454.7696]$ & $1370.7836_{-0.0062}^{+0.0072}$ \\
\hline$\sqrt{e} \sin \omega$ & $\mathcal{F}[0]$ & 0 \\
\hline$\sqrt{e} \cos \omega$ & $\mathcal{F}[0]$ & 0 \\
\hline Scaled semimajor axis $a / R_{\star}$ & $\mathcal{U}[1.1,100]$ & $47.2_{-3.8}^{+2.1}$ \\
\hline Scaled planet radius $R_{\mathrm{p}} / R_{\star}$ & $\mathcal{U}[0,0.06]$ & $0.03165_{-0.00061}^{+0.00072}$ \\
\hline Impact parameter, $b$ & $\mathcal{U}[0,1]$ & $0.3_{-0.19}^{+0.18}$ \\
\hline Parametrized limb-darkening coefficient $q_{1}$ & $\mathcal{U}[0,1]$ & $0.59_{-0.27}^{+0.28}$ \\
\hline Parametrized limb-darkening coefficient $q_{2}$ & $\mathcal{U}[0,1]$ & $0.22_{-0.15}^{+0.25}$ \\
\hline \multicolumn{3}{|l|}{ Derived parameters } \\
\hline Planet radius $\left(R_{\oplus}\right)$ & - & $6.71 \pm 0.38$ \\
\hline Orbit eccentricity $e$ & - & 0 \\
\hline semi-major axis $a(\mathrm{AU})$ & - & $0.423_{-0.037}^{+0.031}$ \\
\hline Orbit inclination $i(\mathrm{deg})$ & - & $89.64_{-0.27}^{+0.24}$ \\
\hline Stellar density $\rho_{\star}$ (from LC) & - & $0.283_{-0.064}^{+0.039}$ \\
\hline $\begin{array}{l}\text { Equilibrium temperature (albedo }=0) T_{\mathrm{eq}} \\
(K)\end{array}$ & - & $610_{-21}^{+28}$ \\
\hline Insolation $F_{\mathrm{p}}\left(F_{\oplus}\right)$ & - & $23.1_{-3.1}^{+4.6}$ \\
\hline
\end{tabular}

\section{RESULTS AND DISCUSSION}

Having established that the transits are almost definitely caused by a planet, we proceed to derive the parameters of the planet by detailed modelling of the TESS light curve, combined with the stellar parameters derived in Section 4.

\subsection{Transit modelling}

The TOI 813 transits were modelled using the open source software pyaneti (Barragán et al. 2019), which was previously used for the analysis of other exoplanets discovered by TESS (e.g. Gandolfi et al. 2018; Esposito et al. 2019). We first isolated and flattened each transit using exotrending (Barragán \& Gandolfi 2017) as described by Barragán et al. (2018). When modelling the Sector 2 transit, the variations in the transit model during each 30-min exposure must be accounted for explicitly (Kipping 2010); we thus computed the model at 3-min intervals and integrated it to 30-min sampling before comparing it to the observations. Details of the fitted parameters and the priors used are given in Table 3. Note that we kept the eccentricity fixed to zero for the fiducial analysis, and used the $\left(q_{1}, q_{2}\right)$ limb-darkening parametrization to efficiently sample the range of physical solutions (see Kipping 2013). The parameter space was explored using an MCMC approach with 500 chains. Once the chains had converged, we used the last 5000 iterations with a thinning factor of 10 to create posterior distributions based on 250000 sampled points for each fitted parameter.

We inspected the posterior distributions visually and found them to be smooth and unimodal for all fitted parameters. We also inspected the residuals between the model and data and conclude that they show no evidence of correlated noise. We also find no evidence of enhanced scatter in the residuals. The phase-folded data and best-fitting model are shown in Fig. 9, and the median and

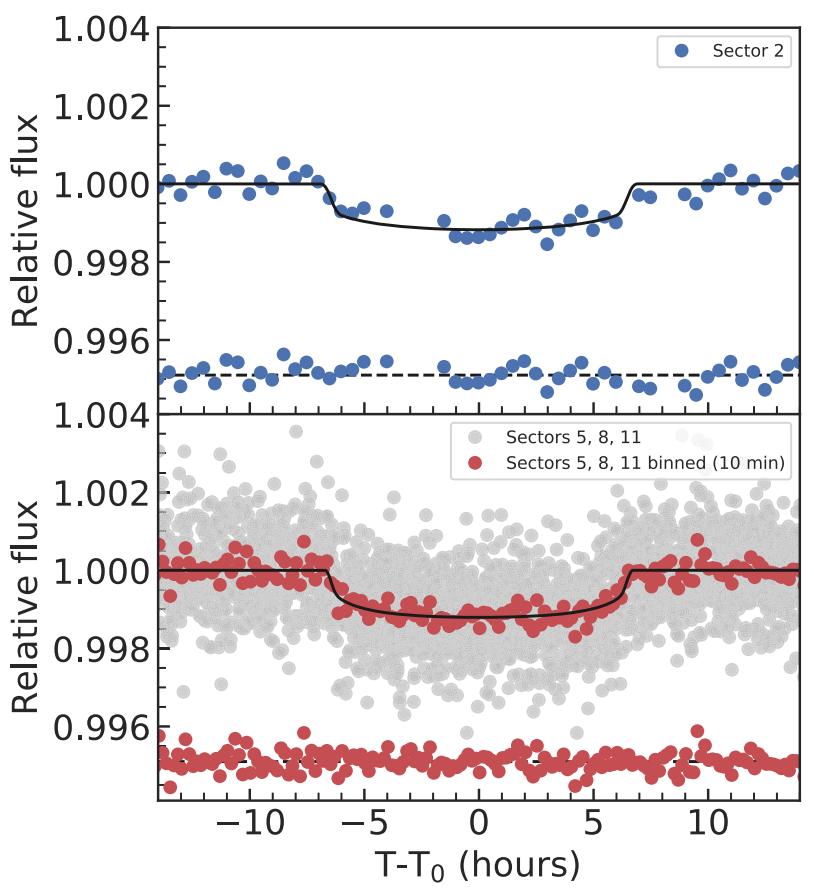

Figure 9. Phase-folded TESS light curve of TOI $813 \mathrm{~b}$ with the best-fitting transit model overplotted and residuals.

68.3 per cent central interval of the posterior distribution for each parameter are listed in Table 3.

After fitting all four transits simultaneously, we fitted each of the four transits in turn, keeping all parameters fixed except 
for the time of transit centre. The resulting transit times were (in units of BJD - 2457000 ) $1370.800_{-0.011}^{+0.013} \mathrm{~d}, 1454.6767_{-0.0081}^{+0.0077} \mathrm{~d}$, $1538.5625_{-0.0039}^{+0.0043} \mathrm{~d}$, and $1622.4580_{-0.0041}^{+0.004} \mathrm{~d}$. The larger uncertainty on the time of the first transit is due to the longer cadence of the Sector 2 observations. We note that these values are consistent with a constant period.

The stellar density derived from the transit modelling $\left(0.283_{-0.064}^{+0.039} \mathrm{~g} \mathrm{~cm}^{-3}\right)$ is in good agreement with the value derived from the spectral analysis, $\left(0.254_{-0.037}^{+0.046} \mathrm{~g} \mathrm{~cm}^{-3}\right)$ implying that the assumption of zero eccentricity is justified, or at the very least consistent with the available data. We did however test the effect of relaxing this assumption, by repeating the transit modelling with a free eccentricity. The model was run with uniform priors over the interval $[-1,1]$ for the parameters $\sqrt{e} \sin \omega$ and $\sqrt{e} \cos \omega$ (Anderson et al. 2011), and a prior on $a / R_{\star}$ that was based on the stellar density derived in Section 4.2 and on Kepler's third law. This resulted in fitted values of $\sqrt{e} \sin \omega=0.00 \pm 0.15$ and $\sqrt{e} \cos \omega=0.04_{-0.22}^{+0.20}$, which translate to an eccentricity of $e=0.05_{-0.03}^{+0.06}$, with an upper limit of $e<0.2$ at the 99 per cent confidence level.

Data from the NASA Exoplanet Archive (Akeson et al. 2013) show that single planetary systems with orbital periods within 15 per cent of that of TOI $813 \mathrm{~b}$ have a median eccentricity of 0.23 . The apparently low eccentricity of TOI $813 \mathrm{~b}$ is characteristic of planets in multiple transiting systems (Van Eylen et al. 2019). This result, together with TOI 813 b's long orbital period and the high fraction of main-sequence stars that host nearly coplanar multiplanet systems (e.g. Rowe et al. 2014), motivated us to search for additional transits in the TESS light curve, as described in Section 3.4, but none were found. However, a large fraction of planets smaller than $\sim 5 R_{\oplus}$ and/or on periods longer than $\sim 60 \mathrm{~d}$ would have been missed by such a search, as would any planets or orbits that were not coplanar enough with that of TOI $813 \mathrm{~b}$ to transit. The possibility that TOI 813 is a multiplanet system remains open, and merits future exploration (e.g. using RV measurements).

\subsection{Planets around subgiant stars}

TOI $813 \mathrm{~b}$ is in orbit around a subgiant host. The subgiant phase in some respects mimics the pre-main sequence phase in reverse: the same star-planet interaction mechanisms - tides, orbital migration, stellar heating, and photoevaporation - are at play, but their effect is increasing, rather than decreasing, with time. Using a MIST Version-1.2 stellar evolution track (Choi et al. 2016), with input stellar parameters as given in Table 1 and a rotation velocity of $0 \mathrm{~m} \mathrm{~s}^{-1}$, we estimate the main sequence lifetime of TOI 813 to be $\sim 3.45 \mathrm{Gyr}$. This means that, at the current age of $3.73 \pm 0.62 \mathrm{Gyr}$, the target has only recently left the main sequence and is still in the process of entering what will become a very rapid period of evolution.

TOI $813 \mathrm{~b}$ is relatively distant from its host star (scaled semimajor axis $a / R_{\star}=47.2_{-3.8}^{+2.1}$ ) and is not expected to interact with it very strongly. Specifically, there is no reason to believe that the orbit of TOI $813 \mathrm{~b}$ has been affected significantly by tidal interaction during the main-sequence lifetime of the star (e.g. Lanza \& Mathis 2016), or that the planet's size and composition have been altered in a major way by stellar irradiation (e.g. Vazan et al. 2013). However, as the star evolves further along the red giant branch the stellar luminosity and radius will increase. Using the same MIST stellar evolution track as for the analysis outlined above, we estimate that the star will reach a maximum radius of 0.76 au on the red giant branch. Furthermore, the model showed that in the absence of orbital evolution of the planet, TOI $813 \mathrm{~b}$ will be engulfed at an age of $\sim 4.66 \mathrm{Gyr}$.

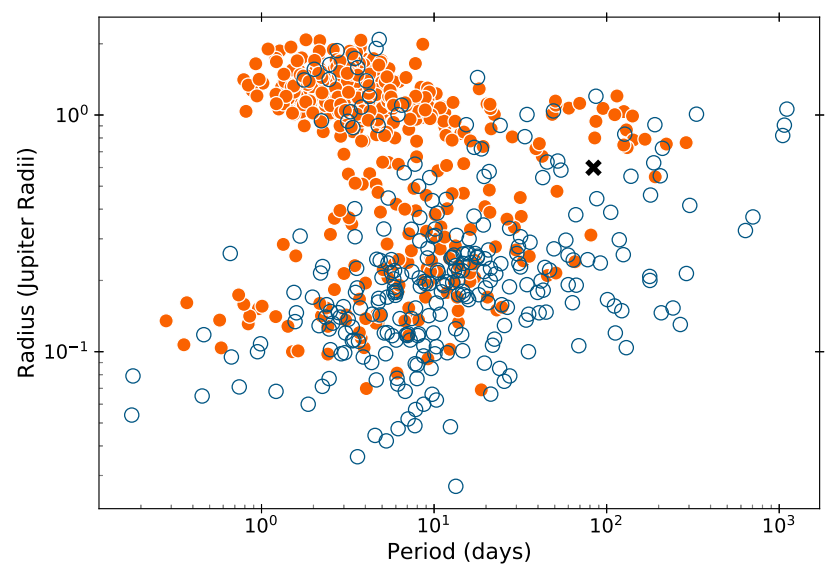

Figure 10. A log-log plot of the period versus radius of transiting exoplanets as they appear in the TEPCAT catalogue (Southworth 2011). The filled orange circles represent planets that have an at least 3-sigma mass measurement. The black cross denotes the properties of TOI $813 \mathrm{~b}$.

While the radius of the star increased on the red giant branch, the mass can be assumed to remain near constant. We therefore estimate that the star's main sequence radius was $\sim 1.1 R_{\odot}$ using a mass-radius relation for main sequence stars $R \propto M^{3 / 7}$ (derived from mass/radius measurements of 190 binary systems by Torres, Andersen \& Giménez (2010b). This implies that the transit of TOI $813 \mathrm{~b}$ is already $\sim 4$ times shallower than it used to be, highlighting the difficulties associated with detecting transiting planets around evolved host stars.

Even though the number of confirmed planets around evolved stars remains small, there is growing evidence that these systems' properties differ from those of their main sequence counterparts (e.g. Johnson et al. 2007; Luhn et al. 2019). We explored this by evaluating the properties of planets around subgiant stars in the NASA Exoplanet Archive, where the subgiants were identified using the data-driven boundaries in effective temperature and surface gravity outlined in Huber et al. (2016).

Out of the 4043 confirmed exoplanets listed in the archive, as of 2019 September, 703 have a subgiant host, 344 of which were discovered using the transit method. Out of these, only 130 have a mass measurement with an accuracy better than $3 \sigma$. Approximately 42 percent of these with measured semimajor axes lie beyond $0.5 \mathrm{AU}$ of the host star, compared to $\sim 15$ per cent for planets around dwarf stars, highlighting that detected planets around subgiants tend to have longer orbital periods. We found no significant differences in the eccentricities of the planets around subgiants compared to those around dwarfs in the sample.

Other validated planets in orbit around bright subgiant host stars observed by TESS include HD 1397b (Brahm et al. 2019) and HD 221416 (Huber et al. 2019).

\subsection{Follow-up prospects}

TOI $813 \mathrm{~b}$ is of particular interest due to its long orbital period compared to other targets found by TESS. Accurate mass measurements of long-period transiting planets around bright stars are rare, as shown in Fig. 10, as are detailed studies of planets orbiting around evolved stars. Additional observations of this target can therefore help to explore a relatively under explored region of parameter space. 
Future RV measurements will allow us to constrain the mass, and therefore density, of TOI $813 \mathrm{~b}$. Using forecaster (Chen \& Kipping 2017) we estimate the planet to have a mass of $42_{-19}^{+49} M_{\oplus}$ corresponding to a Doppler semi-amplitude of $5.6_{-2.6}^{+5.3} \mathrm{~m} \mathrm{~s}^{-1}$, and thus making it a good target for RV follow-up with state-of-theart spectrographs in the Southern hemisphere such as HARPS. These predictions are consistent with the upper mass limit derived in Section 5.2.

Precise RV measurements during the transit may also reveal small deviations from the Keplerian fit in the RV curve (the so-called Rossiter-McLaughlin, or RM, effect; McLaughlin 1924; Rossiter 1924). The RM effect can be used to estimate the project angle between host star's spin axis and the normal to the orbital plane of the planet (e.g. Schneider 2000). While planetary migration through the disc should preserve, or even reduce, the primordial spin-orbit alignment, effects such as planet-planet scattering and Lidov-Kozai resonance (Kozai 1962; Lidov 1962) should result in a misalignment over time (e.g. Deeg et al. 2009; Storch, Lai \& Anderson 2017). Measuring the RM effect can therefore help constrain the dynamical history of the system. For TOI-813b, we estimate the amplitude of the RM effect (which scales with the projected stellar equatorial rotational velocity $\left(v \sin i_{\star}\right.$; Winn 2010), to be around $7.55_{-0.37}^{+0.32} \mathrm{~m} \mathrm{~s}^{-1}$ and therefore detectable using instruments such as HARPS. The RM effect combined with the stellar rotation period would allow us to measure the true $3 \mathrm{D}$ obliquity of the system. Unfortunately, we were unable to determine a rotation period for TOI 813 from the Lomb-Scargle periodogram (see Section 4).

Long-period transiting planets such as TOI $813 \mathrm{~b}$ are also potentially interesting targets for atmospheric follow up. TOI $813 \mathrm{~b}$ has relatively low insolation $\left(23.1_{-3.1}^{+4.6} \mathrm{~F}_{\oplus}\right)$ and equilibrium temperature $\left(610_{-21}^{+28} \mathrm{~K}\right.$, estimated using the Stefan-Boltzmann law and assuming an albedo of 0 ), and measuring its transmission spectrum would provide useful clues as to the atmospheric conditions in a relatively cool giant planet. To evaluate the feasibility of such an observation, we used the transmission spectroscopy metric (TSM) proposed by Kempton et al. (2018). The TSM can be used as an approximation of the expected SNR for a $10 \mathrm{~h}$ observation with JWST/NRIRISS based on the assumption of a cloud-free atmosphere. To calculate it for TOI $813 \mathrm{~b}$, we assumed a mass of $42 M_{\oplus}$ and a mean molecular weight of 2.3 . The resulting TSM of $\sim 20$ is relatively low, as expected (cooler atmospheres are less puffy), but this is to some extent compensated by the relatively long duration of the transit.

\subsection{Prospects for PHT}

This is the first validated TESS exoplanet found by citizen scientists taking part in the PHT project, but many more possible discoveries are actively being followed up. PHT discovers planet candidates through two distinct routes: about 15 percent are brought to the attention of the science team via the Talk discussion boards, while the rest are identified by the main PHT TESS pipeline, which combines the classifications of multiple volunteers for each light curve, using a density based clustering algorithm (Eisner et al. in preparation). Based on our preliminary findings for the first nine TESS observation Sectors, we find about five high-quality candidates per sector, which pass all the light curve based vetting tests discussed in Section 3.3 but were not found by the SPOC and QLP pipelines. Extrapolating to the 2-yr nominal TESS missions, we thus expect PHT to find over 100 new candidates over all.

About two-thirds of our candidates so far are long-period, exhibiting only a single-transit event in the TESS data. These are much more challenging to validate statistically, and are particularly challenging to follow up, so the few high-latitude systems that were observed by TESS long enough to display multiple transits are particularly valuable. Interestingly, several of our other early candidates are listed in the TIC as subgiant stars. These all have long periods and hence long durations, which may explain why more of them were missed by the standard pipelines. If this trend is confirmed, the detection of planets around evolved stars, particularly those where mass measurements are feasible, will be one of the lasting contributions of the PHT project.

\section{CONCLUSIONS}

We report on the discovery and validation of the first TESS planet that was found through a citizen science campaign. The signal was initially discovered by Planet Hunters TESS volunteers as a single transit event in Sector 5 target pixel light curve (2-min cadence). Three additional transits were later found in the target pixel files of sectors 8 and 11 and in the full frame images (30-min cadence) of Sector 2 .

The candidate passed all of our light curve based vetting checks, including, but not limited to, odd-even transit depth comparison, checks for systematic effects, searches for secondary eclipses, and pixel-level centroid analysis to search for blends. Further false positive scenarios, including blended eclipsing binaries, were ruled out with the aid of speckle imaging. These observations, obtained with Gemini and SOAR, showed no signs of stellar companions down to a magnitude difference of 4-5 within 1.17 arcsec and down to a magnitude difference of seven within 3 arcsec of the target. Additionally, we obtained reconnaissance and high-resolution spectra of the star in order to refine the stellar parameter and allowing us to statistically validate the planet, with a false positive probability of 0.003 , using vespa. Finally we determined an upper mass limit of the candidate using RV observations obtained with the CHIRON spectrograph. The upper limit of $2 M_{\text {Jup }}$ (at the 99 per cent credible interval) is consistent with a planetary companion scenario.

A BLS search, carried out on the detrended light curve, did not reveal any additional transit signals. We therefore carried out injection and recovery tests to quantify the detectability of potential further planets in the system. The results showed that we were able to recover $>80$ percent of the injected planets larger than $\sim 5 R_{\oplus}$ with periods $<30$ and $\sim 50$ percent of the simulated planets with periods $<60 \mathrm{~d}$, meaning that we are not able to rule out the presence of additional planets inside the orbit of TOI $813 \mathrm{~b}$.

Detailed modelling of the transits yield that the planet has an orbital period of $83.8911_{-0.0031}^{+0.0027} \mathrm{~d}$, a planet radius of $6.71 \pm 0.38 R_{\oplus}$, and a semimajor axis of $0.423_{-0.037}^{+0.031}$ AU. Furthermore, the planet is in orbit around a bright $(V=10.3 \mathrm{mag}) \operatorname{subgiant}\left(R_{\star}=1.94 R_{\odot}, M_{\star}=\right.$ $\left.1.32 M_{\odot}\right)$ star which is in the process of evolving away from the main sequence and on to the red giant branch. Stellar evolutionary tracks showed that the expanding stellar radius will reach the current semimajor axis of TOI $813 \mathrm{~b}$ in $\sim 0.93 \mathrm{Gyr}$.

The planet's relatively long orbital period together with the evolved nature of the host star places TOI $813 \mathrm{~b}$ in a relatively under explored region of parameter space and is therefore an exciting target for follow-up observations. Based on the stellar brightness $(V=10.3 \mathrm{mag})$ and expected planetary mass $\left(42_{-19}^{+49} M_{\oplus}\right)$, we estimate that TOI $813 \mathrm{~b}$ induces a reflex motion with a Doppler semi-amplitude of $\sim 6 \mathrm{~m} \mathrm{~s}^{-1}$, making this a promising candidate for which we can obtain a precise mass measurement.

Over the 2-yr TESS mission we expect the Planet Hunters TESS project to find over 100 new planet candidates in the 2-min cadence light curves alone. We anticipate that some of these long-period 
planet candidates may be re-detected in the TESS extended mission, yielding precise orbital period measurements and paving the way for more detailed studies.

\section{ACKNOWLEDGEMENTS}

We like to thank all of the volunteers who participated in the Planet Hunters TESS project, as without them this work would not have been possible.

NLE thanks the Large Synoptic Survey Telescope Corporation (LSSTC) Data Science Fellowship Program, which is funded by LSSTC, NSF Cybertraining Grant number 1829740, the Brinson Foundation, and the Moore Foundation; her participation in the program has benefited this work. NLE also acknowledges funding from the Science and Technology Funding Council (STFC) Grant Code ST/R505006/1. AV's work was performed under contract with the California Institute of Technology/Jet Propulsion Laboratory funded by NASA through the Sagan Fellowship Program executed by the NASA Exoplanet Science Institute. MHK thanks Allan R. Schmitt and Troy Winarski for making their light curve examining softwares LCTOOLS and AKO-TPF freely available. MES was supported by Gemini Observatory and also in part by Queen's University Belfast. AZ acknowledges support from an Australian Government Research Training Program (RTP) Scholarship. OB and SA acknowledge support from the UK Science and Technology Facilities Council (STFC) under grants ST/S000488/1 and ST/R004846/1. We would also like to thank the Zorro team and for their help with obtaining speckle images that allowed us to validate this planet and Andy Stephens for his insight into Zorro/' Alopeke. LMFT would like to thank the CONACyT for its support through the grant CVU 555458.

This paper includes data collected with the TESS mission, obtained from the MAST data archive at the Space Telescope Science Institute (STScI). Funding for the TESS mission is provided by the NASA Explorer Program. STScI is operated by the Association of Universities for Research in Astronomy, Inc., under NASA contract NAS 5-26555.

This research has made use of the NASA Exoplanet Archive and the Exoplanet Follow-up Observation Program website, which are operated by the California Institute of Technology, under contract with the National Aeronautics and Space Administration under the Exoplanet Exploration Program.

Resources supporting this work were provided by the NASA High-End Computing (HEC) Program through the NASA Advanced Supercomputing (NAS) Division at Ames Research Center for the production of the SPOC data products.

This work is based in part on observations from Director's Discretionary program GS-2019A-DD-109 at the Gemini Observatory, which is operated by the Association of Universities for Research in Astronomy, Inc., under a cooperative agreement with the NSF on behalf of the Gemini partnership: the National Science Foundation (United States), National Research Council (Canada), Comisión Nacional de Investigación Científica y Tecnológica (CONICYT, Chile), Ministerio de Ciencia, Tecnología e Innovación Productiva (Argentina), Ministério da Ciência, Tecnologia e Inovação (Brazil), and Korea Astronomy and Space Science Institute (Republic of Korea).

This paper is based on observations obtained at the Southern Astrophysical Research (SOAR) telescope, which is a joint project of the Ministério da Ciência, Tecnologia, Inovações e Comunicações (MCTIC) do Brasil, the U.S. National Optical Astronomy Observatory (NOAO), the University of North Carolina at Chapel Hill (UNC), and Michigan State University (MSU).
NLE and OB wish to thank the charming local café in Oxford, where TOI-813b was first identified as a high-priority PHT candidate and affectionately dubbed Nora- $1 b$, and where most of this paper was written.

Based on observations made with the ESO-3.6m telescope at $\mathrm{La}$ Silla Observatory (Chile) under program 1102.C-0923.

\section{REFERENCES}

Aigrain S., Irwin M., 2004, MNRAS, 350, 331

Akeson R. L. et al., 2013, PASP, 125, 989

Anderson D. R. et al., 2011, ApJ, 726, L19

Baranne A. et al., 1996, A\&AS, 119, 373

Barclay T., Pepper J., Quintana E. V., 2018, ApJS, 239, 2

Barragán O., Gandolfi D., 2017, Exotrending, record ascl:1706.001

Barragán O. et al., 2018, A\&A, 612, A95

Barragán O., Gandolfi D., Antoniciello G., 2019, MNRAS, 482, 1017

Blanco-Cuaresma S., Soubiran C., Heiter U., Jofré P., 2014, A\&A, 569, A111

Borucki W. J. et al., 2012, ApJ, 745, 120

Boyajian T. S. et al., 2016, MNRAS, 457, 3988

Brahm R. et al., 2019, AJ, 158, 45

Bressan A., Marigo P., Girardi L., Salasnich B., Dal Cero C., Rubele S., Nanni A., 2012, MNRAS, 427, 127

Bruntt H. et al., 2010, MNRAS, 405, 1907

Chen J., Kipping D., 2017, ApJ, 834, 17

Childress M. J., Vogt F. P. A., Nielsen J., Sharp R. G., 2014, Ap\&SS, 349, 617

Choi J., Dotter A., Conroy C., Cantiello M., Paxton B., Johnson B. D., 2016, ApJ, 823, 102

Christiansen J. L. et al., 2018, AJ, 155, 57

Deeg H. J. et al., 2009, A\&A, 506, 343

Díaz R. F., Almenara J. M., Santerne A., Moutou C., Lethuillier A., Deleuil M., 2014, MNRAS, 441, 983

Dopita M., Hart J., McGregor P., Oates P., Bloxham G., Jones D., 2007, Ap\&SS, 310, 255

Doyle A. P., Davies G. R., Smalley B., Chaplin W. J., Elsworth Y., 2014, MNRAS, 444, 3592

Esposito M. et al., 2019, A\&A, 623, A165

Fausnaugh M., Huang X., Glidden A., Guerrero N., TESS Science Office, 2018, American Astronomical Society Meeting Abstracts \#231. p. 439.09

Feinstein A. D. et al., 2019, PASP, 131, 94502

Fischer D. A. et al., 2012, MNRAS, 419, 2900

Foreman-Mackey D., Hogg D. W., Lang D., Goodman J., 2013, PASP, 125 , 306

Foreman-Mackey D., Morton T. D., Hogg D. W., Agol E., Schölkopf B., 2016, AJ, 152, 206

Fridlund M. et al., 2017, A\&A, 604, A16

Gaia Collaboration et al.2018, A\&A, 616, A1

Gandolfi D. et al., 2018, A\&A, 619, L10

Gray R. O., Corbally C. J., 1994, AJ, 107, 742

Howell S. B., Everett M. E., Sherry W., Horch E., Ciardi D. R., 2011, AJ, 142,19

Huang C. X. et al., 2018, ApJ, 868, L39

Huber D. et al., 2016, ApJS, 224, 2

Huber D. et al., 2019, AJ, 157, 245

Høg E. et al., 2000, A\&A, 355, L27

Jenkins J. M. et al., 2016, in Chiozzi G., Guzman J. C., eds, Proc. SPIE Conf. Ser. Vol. 9913, Software and Cyberinfrastructure for Astronomy IV. SPIE, Bellingham, p. 99133E

Johnson J. A. et al., 2007, ApJ, 665, 785

Kanodia S., Wright J., 2018, Res. Notes Am. Astron. Soc., 2, 4

Kempton E. M.-R. et al., 2018, PASP, 130, 114401

Kipping D. M., 2010, MNRAS, 408, 1758

Kipping D. M., 2013, MNRAS, 435, 2152

Kipping D. M., Schmitt A. R., Huang X., Torres G., Nesvorný D., Buchhave L. A., Hartman J., Bakos G. Á., 2015, ApJ, 813, 14 
Kovács G., Zucker S., Mazeh T., 2002, A\&A, 391, 369

Kozai Y., 1962, AJ, 67, 591

Kreidberg L., 2015, PASP, 127, 1161

Kurucz R. L., 2013, ATLAS12, record ascl:1303.024

Lanza A. F., Mathis S., 2016, Celest. Mech. Dyn. Astron., 126, 249

Li J., Tenenbaum P., Twicken J. D., Burke C. J., Jenkins J. M., Quintana E. V., Rowe J. F., Seader S. E., 2019, PASP, 131, 024506

Lidov M. L., 1962, Planet. Space Sci., 9, 719

Lintott C. et al., 2011, MNRAS, 410, 166

Lintott C. J. et al., 2008, MNRAS, 389, 1179

Lintott C. J. et al., 2013, AJ, 145, 151

Livingston J. H. et al., 2018, AJ, 156, 277

Lomb N. R., 1976, Ap\&SS, 39, 447

Luhn J. K., Bastien F. A., Wright J. T., Johnson J. A., Howard A. W., Isaacson H., 2019, AJ, 157, 149

Matson R. A., Howell S. B., Ciardi D. R., 2019, AJ, 157, 211

Mayor M. et al., 2003, Messenger, 114, 20

McLaughlin D. B., 1924, ApJ, 60, 22

Montet B. T. et al., 2015, ApJ, 809, 25

Morton T. D., 2012, ApJ, 761, 6

Morton T. D., 2015, VESPA: False positive probabilities calculator, Astrophysics Source Code Library, record ascl:1503.011

Morton T. D., Bryson S. T., Coughlin J. L., Rowe J. F., Ravichandran G., Petigura E. A., Haas M. R., Batalha N. M., 2016, ApJ, 822, 86

Munari U. et al., 2014, AJ, 148, 81

Pearson K. A., Palafox L., Griffith C. A., 2018, MNRAS, 474, 478

Pecaut M. J., Mamajek E. E., 2013, ApJS, 208, 9

Persson C. M. et al., 2018, A\&A, 618, A33

Piskunov N., Valenti J. A., 2017, A\&A, 597, A16

Rappaport S. et al., 2019, MNRAS, 488, 2455

Ricker G. R. et al., 2015, J. Astron. Telesc. Instrum. Syst., 1, 014003

Rossiter R. A., 1924, ApJ, 60, 15

Rowe J. F. et al., 2014, ApJ, 784, 45

Rowe J. F. et al., 2015, ApJS, 217, 16

Ryabchikova T., Piskunov N., Kurucz R. L., Stempels H. C., Heiter U., Pakhomov Y., Barklem P. S., 2015, Phys. Scr., 90, 054005

Santerne A. et al., 2015, MNRAS, 451, 2337

Scargle J. D., 1982, ApJ, 263, 835

Schlegel D. J., Finkbeiner D. P., Davis M., 1998, ApJ, 500, 525

Schmitt J. R. et al., 2014a, AJ, 148, 28

Schmitt J. R. et al., 2014b, ApJ, 795, 167

Schneider J., 2000, in Griffith C. A., Marley M. S., eds, ASP Conf. Ser. Vol. 212, From Giant Planets to Cool Stars. Astron. Soc. Pac., San Francisco, p. 284

Schwamb M. E. et al., 2012, ApJ, 754, 129

Schwamb M. E. et al., 2013, ApJ, 768, 127

Skrutskie M. F. et al., 2006, AJ, 131, 1163

Smith J. C. et al., 2012, PASP, 124, 1000

Southworth J., 2011, MNRAS, 417, 2166

Stassun K. G., Torres G., 2016, AJ, 152, 180

Stassun K. G., Torres G., 2018, ApJ, 862, 61

Stassun K. G., Collins K. A., Gaudi B. S., 2017, AJ, 153, 136

Stassun K. G., Corsaro E., Pepper J. A., Gaudi B. S., 2018, AJ, 155, 22

Stassun K. G. et al., 2019, AJ, 158, 138

Storch N. I., Lai D., Anderson K. R., 2017, MNRAS, 465, 3927

Stumpe M. C. et al., 2012, PASP, 124, 985

Tokovinin A., 2018, PASP, 130, 035002

Tokovinin A., Fischer D. A., Bonati M., Giguere M. J., Moore P., Schwab C., Spronck J. F. P., Szymkowiak A., 2013, PASP, 125, 1336

Torres G., Andersen J., Giménez A., 2010a, A\&AR, 18, 67

Torres G., Andersen J., Giménez A., 2010b, A\&AR, 18, 67

Twicken J. D. et al., 2018, PASP, 130, 064502

Valenti J. A., Piskunov N., 1996, A\&AS, 118, 595

Van Eylen V. et al., 2019, AJ, 157, 61

Vanderburg A. et al., 2019, ApJ, 881, L19

Vazan A., Kovetz A., Podolak M., Helled R., 2013, MNRAS, 434, 3283

Wang J. et al., 2013, ApJ, 776, 10

Wang J. et al., 2015, ApJ, 815, 127
Winn J. N., 2010, Exoplanet Transits and Occultations. University of Arizona Press, Tucson, AZ, p. 55

Wright E. L. et al., 2010, 140, AJ, 1868

Yee S. W., Petigura E. A., von Braun K., 2017, ApJ, 836, 77

Ziegler C., Tokovinin A., Briceno C., Mang J., Law N., Mann A. W., 2020, AJ, 159, 19

Zink J. K. et al., 2019, Res. Notes Am. Astron. Soc., 3, 43

Zucker S., Giryes R., 2018, AJ, 155, 147

${ }^{1}$ Department of Physics, University of Oxford, Keble Road, Oxford OX1 $3 R H, U K$

${ }^{2}$ Department of Physics and Astronomy, Louisiana State University, Baton Rouge, LA 70803 USA

${ }^{3}$ Cerro Tololo Inter-American Observatory, Casilla 603, La Serena 1700000, Chile

${ }^{4}$ Department of Physics, University of Warwick, Gibbet Hill Road, Coventry CV4 7AL, UK

${ }^{5}$ Centre for Exoplanets and Habitability, University of Warwick, Gibbet Hill Road, Coventry CV4 7AL, UK

${ }^{6}$ NASA Ames Research Center, Moffett Field, CA 94035, USA

${ }^{7}$ Department of Astronomy and Astrophysics, University of Chicago, 5640 S. Ellis Ave, Chicago, IL 60637, USA

${ }^{8}$ Departamento de Astronomía, Universidad de Guanajuato, Callejón de Jalisco S/N, Col. Valenciana CP, 36023 Guanajuato, Gto, México

${ }^{9}$ Department of Space, Earth and Environment, Chalmers University of Technology, Onsala Space Observatory, SE-439 92 Onsala, Sweden

${ }^{10}$ Leiden Observatory, University of Leiden, PO Box 9513, NL-2300 RA Leiden, the Netherlands

${ }^{11}$ Dipartimento di Fisica, Universitá di Torino, Via P. Giuria 1, I-10125 Torino, Italy

${ }^{12}$ Research School of Astronomy and Astrophysics, Mount Stromlo Observatory, Australian National University, Cotter Road, Weston Creek, ACT 2611, Australia

${ }^{13}$ Department of Physics, and Kavli Institute for Astrophysics and Space Research, Massachusetts Institute of Technology, Cambridge, MA 02139, USA

${ }^{14}$ DTU Space, National Space Institute, Technical University of Denmark, Elektrovej 327, DK-2800 Lyngby, Denmark

${ }^{15}$ Department of Astronomy, The University of Texas at Austin, Austin, TX 78712, USA

${ }^{16}$ Department of Physics and Astronomy, The University of North Carolina at Chapel Hill, Chapel Hill, NC 27599-3255, USA

${ }^{17}$ Australian Astronomical Optics, 105 Delhi Rd, North Ryde, NSW 2113, Australia

${ }^{18}$ Department of Physics and Astronomy, Macquarie University, NSW 2109, Australia

${ }^{19}$ Gemini Observatory, Northern Operations Center, 670 North A'ohoku Place, Hilo, HI 96720, USA

${ }^{20}$ Astrophysics Research Centre, Queen's University Belfast, Belfast BT7 $1 N N, U K$

${ }^{21}$ Department of Physics and Astronomy, Vanderbilt University, 6301 Stevenson Center Ln., Nashville, TN 37235, USA

${ }^{22}$ Department of Physics, Fisk University, 1000 17th Ave. N., Nashville, TN 37208, USA

${ }^{23}$ Laboratoire d'Astrophysique de Marseille, Aix Marseille Univ, CNRS, CNES, 38 rue Frédéric Joliot-Curie, 13388 Marseille Cedex 13, France

${ }^{24}$ Department of Astronomy, Yale University, New Haven, CT 06511, USA

${ }^{25}$ Sydney Institute for Astronomy, School of Physics, University of Sydney, NSW 2006, Australia

${ }^{26}$ CSIRO Astronomy and Space Science, PO Box 76, Epping, NSW 1710, Australia

${ }^{27}$ Dunlap Institute for Astronomy and Astrophysics, University of Toronto, 50 St. George Street, Toronto, Ontario M5S 3H4, Canada

${ }^{28}$ Planet Hunters TESS, Citizen Scientist

This paper has been typeset from a $\mathrm{T}_{\mathrm{E}} \mathrm{X} / \mathrm{ET} \mathrm{E} \mathrm{X}$ file prepared by the author. 